\title{
A MOLECULAR MECHANICS STUDY OF COPPER(II) CATALYSED ASYMMETRIC DIELS-ALDER REACTIONS
}

\author{
Robert J. Deeth* and Natalie Fey \\ Inorganic Computational Chemistry Group, Department of Chemistry, University of Warwick, \\ Coventry, CV4 7AL, U. K.
}

SUPPORTING INFORMATION 
Table S1: Parameters Added to MMFF94 Force Field in MOE 2001.01

Atom Type

\begin{tabular}{|l|l|l|l|}
\hline Type & Symbol & Element & Description \\
\hline type & $\mathrm{Cx}$ & $\mathrm{Si}$ & special carbon type, 5 bonds \\
\hline
\end{tabular}

Type Matching Rules

\begin{tabular}{|l|l|l|}
\hline Symbol & Rule & Comment \\
\hline $\mathrm{CU}+2$ & match '[CuX5]' & allow for dummy bond \\
\hline $\mathrm{O}=$ & match '[OX2][\#29]' & allow for carbonyl $\mathrm{O}$ coordination to $\mathrm{Cu}$ \\
\hline $\mathrm{O}=$ & atom-name 'O=' & override auto-typing \\
\hline $\mathrm{N}=\mathrm{C}$ & match '[NX3][\#29]' & allow for imine $\mathrm{N}$ coordination to $\mathrm{Cu}$ \\
\hline $\mathrm{N}=\mathrm{C}$ & atom-name 'N=C' & override auto-typing \\
\hline $\mathrm{C}$ & atom-name 'Cx' & ensure same $\mathrm{C}$ atom type for all spiro ligands \\
\hline $\mathrm{Cx}$ & atom-name 'SiX' & override auto-typing for special $\mathrm{C}$ \\
\hline $\mathrm{NC}=\mathrm{O}$ & atom-name 'NC=O' & override auto-typing \\
\hline
\end{tabular}

Bond Stretches

\begin{tabular}{|c|c|c|c|c|c|c|c|c|}
\hline \multirow[t]{2}{*}{ Context Code } & \multirow[t]{2}{*}{ T1 } & \multirow[t]{2}{*}{$\mathbf{T} 2$} & \multirow[t]{2}{*}{$\begin{array}{c}\text { Length } \\
/ \AA\end{array}$} & \multicolumn{3}{|c|}{$\begin{array}{l}\text { Force Constants } \\
\text { / kcal mol } \AA^{-1} \AA^{-1}\end{array}$} & \multirow[t]{2}{*}{$\begin{array}{l}\text { bond charge } \\
\text { increment }\end{array}$} & \multirow[t]{2}{*}{ Comment } \\
\hline & & & & k2 & k3 & k4 & & \\
\hline * & $\mathrm{Cx}$ & $\mathrm{C}$ & 1.508 & 306.432 & -612.865 & 715.009 & 0.000 & \multirow{4}{*}{$\begin{array}{l}\text { cf. existing C } \\
\text { parameters }\end{array}$} \\
\hline * & $\mathrm{Cx}$ & $\mathrm{C}=$ & 1.492 & 301.539 & -603.077 & 703.590 & -0.061 & \\
\hline * & $\mathrm{Cx}$ & $\mathrm{HC}$ & 1.093 & 342.991 & -685.982 & 800.313 & 0.000 & \\
\hline$*$ & $\mathrm{Cx}$ & $\mathrm{N}=\mathrm{C}$ & 1.458 & 342.775 & -685.550 & 799.809 & -0.246 & \\
\hline$*$ & $\mathrm{CU}+2$ & $\mathrm{~N}=\mathrm{C}$ & 2.000 & 180.000 & -370.000 & 430.000 & - & \\
\hline$*$ & $\mathrm{CU}+2$ & $\mathrm{O}=$ & 2.000 & 180.000 & -370.000 & 430.000 & - & \\
\hline * & $\mathrm{CU}+2$ & $\mathrm{Cx}$ & 3.400 & 0.001 & 0.000 & 0.000 & 0.000 & dummy bond \\
\hline
\end{tabular}

\begin{tabular}{|c|c|c|c|c|c|c|c|c|}
\hline \multicolumn{9}{|l|}{ Angle Bends } \\
\hline \multirow[t]{2}{*}{ Context Code } & \multirow[t]{2}{*}{ T1 } & \multirow[t]{2}{*}{$\mathbf{T 2}$} & \multirow[t]{2}{*}{ T3 } & \multirow[t]{2}{*}{ Angle $/^{\circ}$} & \multicolumn{3}{|c|}{$\begin{array}{l}\text { Force Constants } \\
/ \mathrm{kcal} \mathrm{mol}^{-1} \mathrm{deg}^{-1}\end{array}$} & \multirow[t]{2}{*}{ Comment } \\
\hline & & & & & k2 & k3 & k4 & \\
\hline$*$ & $\mathrm{O}=$ & $\mathrm{C}=$ & $\mathrm{NC}=\mathrm{O}$ & 124.152 & 65.2729 & -26.1790 & 0 & \multirow[t]{13}{*}{ cf. existing $\mathrm{C}$ parameters } \\
\hline$*$ & $\mathrm{C}$ & $\mathrm{N}=\mathrm{C}$ & $\mathrm{C}=$ & 106.409 & 63.1859 & -25.3420 & 0 & \\
\hline$*$ & $\mathrm{C}$ & $\mathrm{C}$ & $\mathrm{C}$ & 109.608 & 61.2428 & -24.5627 & 0 & \\
\hline$*$ & $\mathrm{Cx}$ & $\mathrm{C}=$ & $\mathrm{N}=\mathrm{C}$ & 119.788 & 70.3824 & -28.2283 & 0 & \\
\hline$*$ & $\mathrm{Cx}$ & $\mathrm{C}=$ & $\mathrm{O}$ & 109.716 & 75.0602 & -30.1044 & 0 & \\
\hline$*$ & $*$ & $\mathrm{Cx}$ & $*$ & 108.900 & 0.0000 & -0.0000 & 0 & \\
\hline$*$ & $\mathrm{HC}$ & $\mathrm{Cx}$ & $\mathrm{HC}$ & 108.836 & 37.1343 & -14.8935 & 0 & \\
\hline$*$ & $\mathrm{C}$ & $\mathrm{Cx}$ & $\mathrm{C}$ & 109.608 & 61.2428 & -24.5627 & 0 & \\
\hline$*$ & $\mathrm{HC}$ & $\mathrm{Cx}$ & $\mathrm{C}=$ & 108.385 & 46.7777 & -18.7612 & 0 & \\
\hline$*$ & $\mathrm{C}$ & $\mathrm{Cx}$ & $\mathrm{C}=$ & 107.517 & 55.9173 & -22.4268 & 0 & \\
\hline$*$ & $\mathrm{C}=$ & $\mathrm{Cx}$ & $\mathrm{C}=$ & 111.746 & 70.0946 & -28.1129 & 0 & \\
\hline$*$ & $\mathrm{C}$ & $\mathrm{C}$ & $\mathrm{Cx}$ & 109.608 & 61.2428 & -24.5627 & 0 & \\
\hline$*$ & $\mathrm{HC}$ & $\mathrm{C}$ & $\mathrm{Cx}$ & 110.549 & 45.7702 & -18.3571 & 0 & \\
\hline$*$ & $\mathrm{CU}+2$ & $\mathrm{Cx}$ & $\mathrm{C}=$ & 60.000 & 0.0010 & 0.0000 & 0 & \multirow[t]{5}{*}{ dummy bond terms } \\
\hline$*$ & $\mathrm{CU}+2$ & $\mathrm{Cx}$ & $\mathrm{C}$ & 125.000 & 0.0010 & 0.0000 & 0 & \\
\hline$*$ & $\mathrm{CU}+2$ & $\mathrm{Cx}$ & $\mathrm{HC}$ & 125.000 & 0.0010 & 0.0000 & 0 & \\
\hline$*$ & $\mathrm{Cx}$ & $\mathrm{CU}+2$ & $\mathrm{~N}=\mathrm{C}$ & 46.000 & 15.0000 & 0.0000 & 0 & \\
\hline$*$ & $\mathrm{Cx}$ & $\mathrm{CU}+2$ & $\mathrm{O}=$ & 134.000 & 15.0000 & 0.0000 & 0 & \\
\hline$*$ & $\mathrm{CU}+2$ & $\mathrm{~N}=\mathrm{C}$ & $\mathrm{C}=$ & 120.000 & 62.0000 & -25.0000 & 0 & \\
\hline$*$ & $\mathrm{CU}+2$ & $\mathrm{~N}=\mathrm{C}$ & $\mathrm{C}$ & 120.000 & 62.0000 & -25.0000 & 0 & \\
\hline$*$ & $\mathrm{CU}+2$ & $\mathrm{O}=$ & $\mathrm{C}=$ & 125.000 & 62.0000 & -25.0000 & 0 & \\
\hline$*$ & $\mathrm{~N}=\mathrm{C}$ & $\mathrm{CU}+2$ & $\mathrm{~N}=\mathrm{C}$ & 92.500 & 20.0000 & -2.5000 & 0 & \\
\hline$*$ & $\mathrm{O}=$ & $\mathrm{CU}+2$ & $\mathrm{O}=$ & 84.000 & 20.0000 & -2.5000 & 0 & \\
\hline$*$ & $\mathrm{~N}=\mathrm{C}$ & $\mathrm{CU}+2$ & $\mathrm{O}=$ & 90.000 & 0.0010 & 0.0000 & 0 & \\
\hline
\end{tabular}


Stretch-Bends

\begin{tabular}{|c|c|c|c|c|c|c|}
\hline Context Code & T1 & $\mathbf{T} 2$ & T3 & \multicolumn{2}{|c|}{$\begin{array}{l}\text { Force Constants } \\
\text { / kcal } \mathrm{mol}^{-1} \text { deg }^{-1} \AA^{-1}\end{array}$} & Comment \\
\hline * & $\mathrm{Cx}$ & $\mathrm{C}=$ & $\mathrm{O}$ & 24.32463 & 52.67938 & cf. existing $\mathrm{C}$ parameters \\
\hline * & $\mathrm{HC}$ & $\mathrm{CX}$ & $\mathrm{Hc}$ & 8.27613 & 8.27613 & \\
\hline$*$ & $\mathrm{HC}$ & $\mathrm{Cx}$ & $\mathrm{C}=$ & 11.29872 & 8.27613 & \\
\hline * & $\mathrm{C}$ & $\mathrm{Cx}$ & $\mathrm{C}$ & 14.82507 & 14.82507 & \\
\hline$*$ & $\mathrm{C}$ & $\mathrm{Cx}$ & $\mathrm{C}=$ & 15.18490 & 6.62091 & \\
\hline$*$ & $\mathrm{C}$ & $\mathrm{C}$ & $\mathrm{Cx}$ & 14.82507 & 14.82507 & \\
\hline$*$ & $\mathrm{HC}$ & $\mathrm{C}$ & $\mathrm{Cx}$ & 16.33636 & 5.03765 & \\
\hline
\end{tabular}

Out-of-Plane Interactions (Planar Atoms)

\begin{tabular}{|l|l|l|l|c|}
\hline T1 & T2 & T3 & T4 & $\begin{array}{c}\text { Force Constant koop } \\
\text { / kcal A } \text { mol }^{-1} \text { deg }^{-2}\end{array}$ \\
\hline$*$ & $\mathrm{~N}=\mathrm{C}$ & $*$ & $*$ & 1.43931362 \\
\hline
\end{tabular}

Nonbonded Interactions

\begin{tabular}{|c|c|c|c|c|c|c|}
\hline Type & $\underset{/ \AA}{\operatorname{Radius}^{\mathbf{a}}}$ & $\begin{array}{c}\text { Well Depth } \\
\text { (Energy at Radius) } \\
\text { / kcal } \text { mol }^{-1}\end{array}$ & $\begin{array}{c}\text { Atomic } \\
\text { Polarisability } \\
/ \mathbf{c m}^{-1}\end{array}$ & $\begin{array}{c}\text { Effective No. of } \\
\text { Outer Shell Electrons }\end{array}$ & $\begin{array}{l}\text { Atomic Mass } \\
\text { / amu }\end{array}$ & Comment \\
\hline $\mathrm{Cx}$ & 1.969 & 0.068 & 1.050 & 2.490 & 12.011 & $\begin{array}{l}\text { cf. existing } \\
\text { C parameter }\end{array}$ \\
\hline
\end{tabular}

${ }^{a} / 2$ optimal non-bonded distance between 2 atoms of this type 


\begin{tabular}{|c|c|c|c|c|c|c|c|c|c|c|}
\hline \multicolumn{11}{|c|}{ Proper Torsions } \\
\hline \multirow[t]{2}{*}{ Context Code } & \multirow[t]{2}{*}{ T1 } & \multirow[t]{2}{*}{ T2 } & \multirow[t]{2}{*}{ T3 } & \multirow[t]{2}{*}{ T4 } & \multicolumn{5}{|c|}{$\begin{array}{c}\text { Force Constants } \\
/ \text { kcal mol }^{-1}\end{array}$} & \multirow[t]{2}{*}{ Comment } \\
\hline & & & & & $\mathrm{V} 1 / 2$ & $\mathrm{~V} 2 / 2$ & $\mathrm{~V} 3 / 2$ & V4/2 & $\mathrm{V} 5 / 2$ & \\
\hline * & $\mathrm{Cx}$ & $\mathrm{C}$ & $\mathrm{C}$ & $\mathrm{C}$ & 0.051 & -0.341 & 0.166 & 0.000 & 0.000 & \multirow{21}{*}{$\begin{array}{l}\text { cf. existing C } \\
\text { parameters }\end{array}$} \\
\hline 5 & $\mathrm{Cx}$ & $\mathrm{C}$ & $\mathrm{C}$ & $\mathrm{C}$ & 0.072 & 0.274 & 0.563 & 0.000 & 0.000 & \\
\hline * & $\mathrm{Cx}$ & $\mathrm{C}$ & $\mathrm{C}$ & $\mathrm{HC}$ & 0.320 & 0.315 & 0.132 & 0.000 & 0.000 & \\
\hline * & $\mathrm{Cx}$ & $\mathrm{C}=$ & $\mathrm{O}$ & $\mathrm{C}$ & -0.622 & -2.741 & 0.182 & 0.000 & 0.000 & \\
\hline * & * & $\mathrm{Cx}$ & $\mathrm{C}$ & * & 0.000 & -0.000 & 0.150 & 0.000 & 0.000 & \\
\hline 5 & $*$ & $\mathrm{Cx}$ & $\mathrm{C}$ & $*$ & 0.100 & 0.400 & 0.750 & 0.000 & 0.000 & \\
\hline * & $\mathrm{C}$ & $\mathrm{Cx}$ & $\mathrm{C}$ & $\mathrm{C}$ & 0.051 & -0.341 & 0.166 & 0.000 & 0.000 & \\
\hline 5 & $\mathrm{C}$ & $\mathrm{Cx}$ & $\mathrm{C}$ & $\mathrm{C}$ & 0.072 & 0.274 & 0.563 & 0.000 & 0.000 & \\
\hline * & $\mathrm{C}$ & $\mathrm{Cx}$ & $\mathrm{C}$ & $\mathrm{HC}$ & 0.320 & 0.315 & 0.132 & 0.000 & 0.000 & \\
\hline * & $\mathrm{C}=$ & $\mathrm{Cx}$ & $\mathrm{C}$ & $\mathrm{HC}$ & -0.128 & -0.029 & 0.000 & 0.000 & 0.000 & \\
\hline * & $*$ & $\mathrm{Cx}$ & $\mathrm{C}=$ & $*$ & 0.000 & -0.200 & 0.150 & 0.000 & 0.000 & \\
\hline 2 & $*$ & $\mathrm{Cx}$ & $\mathrm{C}=$ & $*$ & 0.000 & -0.250 & 0.175 & 0.000 & 0.000 & \\
\hline 5 & $*$ & $\mathrm{Cx}$ & $\mathrm{C}=$ & * & 0.000 & -0.000 & 0.000 & 0.000 & 0.000 & \\
\hline * & $*$ & $\mathrm{Cx}$ & $\mathrm{C}=$ & $\mathrm{C}$ & 0.000 & -0.000 & 0.275 & 0.000 & 0.000 & \\
\hline * & $*$ & $\mathrm{Cx}$ & $\mathrm{C}=$ & $\mathrm{HC}$ & 0.000 & -0.100 & 0.350 & 0.000 & 0.000 & \\
\hline * & $\mathrm{C}$ & $\mathrm{Cx}$ & $\mathrm{C}=$ & $\mathrm{O}$ & -0.059 & 0.167 & 0.101 & 0.000 & 0.000 & \\
\hline * & $\mathrm{HC}$ & $\mathrm{Cx}$ & $\mathrm{C}=$ & $\mathrm{O}$ & 0.000 & 0.312 & 0.165 & 0.000 & 0.000 & \\
\hline * & $\mathrm{C}$ & $\mathrm{C}$ & $\mathrm{Cx}$ & $\mathrm{C}=$ & 0.033 & 0.078 & 0.071 & 0.000 & 0.000 & \\
\hline * & $\mathrm{N}=\mathrm{C}$ & $\mathrm{C}=$ & $\mathrm{Cx}$ & $\mathrm{HC}$ & 0.000 & -0.200 & 0.150 & 0.000 & 0.000 & \\
\hline * & $\mathrm{N}=\mathrm{C}$ & $\mathrm{C}=$ & $\mathrm{Cx}$ & $\mathrm{C}$ & 0.000 & -0.200 & 0.150 & 0.000 & 0.000 & \\
\hline * & $\mathrm{O}$ & $\mathrm{C}=$ & $\mathrm{Cx}$ & $\mathrm{C}=$ & 0.000 & -0.200 & 0.150 & 0.000 & 0.000 & \\
\hline * & $\mathrm{N}=\mathrm{C}$ & $\mathrm{C}=$ & $\mathrm{Cx}$ & $\mathrm{C}=$ & 0.000 & -5.000 & 0.000 & 0.000 & 0.000 & $\begin{array}{l}\text { needed to maintain } \\
\text { geometry in presence } \\
\text { of spiro ligands }\end{array}$ \\
\hline * & $\mathrm{HC}$ & C & $\mathrm{N}=\mathrm{C}$ & $\mathrm{C}=$ & 0.102 & 0.168 & -0.176 & 0.000 & 0.000 & \\
\hline * & $\mathrm{CU}+2$ & $\mathrm{~N}=\mathrm{C}$ & $\mathrm{C}=$ & $\mathrm{O}$ & 0.000 & -1.000 & 0.000 & 0.000 & 0.000 & \\
\hline * & $\mathrm{CU}+2$ & $\mathrm{~N}=\mathrm{C}$ & $\mathrm{C}=$ & $\mathrm{HC}$ & 0.000 & -1.000 & 0.000 & 0.000 & 0.000 & \\
\hline * & $\mathrm{CU}+2$ & $\mathrm{~N}=\mathrm{C}$ & $\mathrm{C}$ & $\mathrm{HC}$ & 0.000 & -0.000 & 0.000 & 0.000 & 0.000 & \\
\hline * & $\mathrm{CU}+2$ & $\mathrm{~N}=\mathrm{C}$ & $\mathrm{C}$ & $\mathrm{C}$ & 0.000 & -0.000 & 0.000 & 0.000 & 0.000 & \\
\hline * & $\mathrm{CU}+2$ & $\mathrm{~N}=\mathrm{C}$ & $\mathrm{C}$ & Car & 0.000 & -0.000 & 0.000 & 0.000 & 0.000 & \\
\hline * & $\mathrm{CU}+2$ & $\mathrm{~N}=\mathrm{C}$ & $\mathrm{C}=$ & $\mathrm{Cx}$ & 0.000 & -8.000 & 0.000 & 0.000 & 0.000 & \\
\hline * & $\mathrm{CU}+2$ & $\mathrm{O}=$ & $\mathrm{C}=$ & $*$ & 0.000 & -1.000 & 0.000 & 0.000 & 0.000 & \\
\hline * & $\mathrm{CU}+2$ & $\mathrm{Cx}$ & $\mathrm{C}$ & $\mathrm{HC}$ & 0.000 & -0.000 & 0.150 & 0.000 & 0.000 & \\
\hline * & $\mathrm{CU}+2$ & $\mathrm{Cx}$ & $\mathrm{C}$ & $\mathrm{C}$ & 0.000 & -0.000 & 0.150 & 0.000 & 0.000 & \\
\hline \multirow[t]{4}{*}{ * } & $\mathrm{CU}+2$ & $\mathrm{Cx}$ & $\mathrm{C}$ & $*$ & 0.000 & -0.000 & 0.150 & 0.000 & 0.000 & \\
\hline & $\mathrm{CU}+2$ & Cx & $\mathrm{C}=$ & $*$ & 0.000 & -0.200 & 0.150 & 0.000 & 0.000 & \\
\hline & $\mathrm{CU}+2$ & Cx & $\mathrm{C}=$ & $\mathrm{N}=\mathrm{C}$ & 0.000 & -0.200 & 0.150 & 0.000 & 0.000 & \\
\hline & $\mathrm{CU}+2$ & $\mathrm{Cx}$ & $\mathrm{C}=$ & $\mathrm{O}$ & 0.000 & -0.200 & 0.150 & 0.000 & 0.000 & \\
\hline * & $*$ & $\mathrm{CU}+2$ & $\mathrm{~N}=\mathrm{C}$ & $*$ & 0.000 & -0.000 & 0.000 & 0.000 & 0.000 & \\
\hline * & $\mathrm{N}=\mathrm{C}$ & $\mathrm{CU}+2$ & $\mathrm{~N}=\mathrm{C}$ & $\mathrm{C}=$ & 0.000 & -5.000 & 0.000 & 0.000 & 0.000 & \\
\hline * & $*$ & $\mathrm{CU}+2$ & $\mathrm{O}=$ & $*$ & 0.000 & -0.000 & 0.000 & 0.000 & 0.000 & \\
\hline * & $\mathrm{O}=$ & $\mathrm{CU}+2$ & $\mathrm{O}=$ & $\mathrm{C}=$ & 0.000 & -8.000 & 0.000 & 0.000 & 0.000 & \\
\hline * & $*$ & $\mathrm{CU}+2$ & $\mathrm{Cx}$ & $*$ & 0.000 & 0.000 & 0.000 & 0.000 & 0.000 & \multirow{6}{*}{$\begin{array}{l}\text { centred on dummy } \\
\text { bond }\end{array}$} \\
\hline * & $\mathrm{N}=\mathrm{C}$ & $\mathrm{CU}+2$ & $\mathrm{Cx}$ & $\mathrm{HC}$ & 0.000 & 0.000 & 0.000 & 0.000 & 0.000 & \\
\hline * & $\mathrm{N}=\mathrm{C}$ & $\mathrm{CU}+2$ & $\mathrm{Cx}$ & $\mathrm{C}$ & 0.000 & 0.000 & 0.000 & 0.000 & 0.000 & \\
\hline * & $\mathrm{N}=\mathrm{C}$ & $\mathrm{CU}+2$ & $\mathrm{Cx}$ & $\mathrm{C}=$ & 0.000 & 0.000 & 0.000 & 0.000 & 0.000 & \\
\hline * & $\mathrm{O}=$ & $\mathrm{CU}+2$ & $\mathrm{Cx}$ & $\mathrm{HC}$ & 0.000 & 0.000 & 0.000 & 0.000 & 0.000 & \\
\hline * & $\mathrm{O}=$ & $\mathrm{CU}+2$ & $\mathrm{Cx}$ & $\mathrm{C}$ & 0.000 & 0.000 & 0.000 & 0.000 & 0.000 & \\
\hline * & $\mathrm{O}=$ & $\mathrm{CU}+2$ & $\mathrm{Cx}$ & $\mathrm{C}=$ & 0.000 & -0.844 & 0.000 & 0.000 & 0.000 & $\begin{array}{l}\text { control of ligand plane } \\
\text { twist }\end{array}$ \\
\hline
\end{tabular}


Table S2: Comparison of Selected DFT and MM Calculated Structural Parameters (Sources of

Data: DFT - single optimisation, MM - minima of 100 iteration stochastic conformational searches).

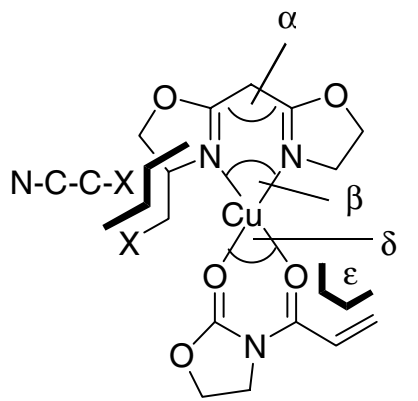

$$
\begin{aligned}
& \alpha=\text { ligand angle }(\mathrm{C}-\mathrm{C}-\mathrm{C}) \\
& \beta=\text { bite angle }(\mathrm{N}-\mathrm{Cu}-\mathrm{N}) \\
& \chi=\text { ligand plane twist }(\mathrm{C}-\mathrm{C} \cdots \mathrm{Cu}-\mathrm{O}, \text { Fig. } 1) \\
& \delta=\text { substrate angle }(\mathrm{O}-\mathrm{Cu}-\mathrm{O}) \\
& \varepsilon=\mathrm{O}=\mathrm{C}-\mathrm{C}=\mathrm{C} \\
& \mathrm{N}-\mathrm{C}-\mathrm{C}-\mathrm{X}=\text { substituent orientation }
\end{aligned}
$$

\begin{tabular}{|c|c|c|c|c|c|c|c|c|}
\hline $\begin{array}{l}\text { Structural } \\
\text { Parameter }\end{array}$ & Method & $5 \mathbf{a}$ & $5 \mathbf{b}$ & $5 \mathrm{c}$ & 5d & $5 e$ & $5 f$ & RMS errors \\
\hline av. $r \mathrm{~N}-\mathrm{Cu} / \AA$ & $\begin{array}{l}\text { DFT } \\
\text { MM }\end{array}$ & $\begin{array}{l}1.99 \\
1.95\end{array}$ & $\begin{array}{l}1.98 \\
1.95\end{array}$ & $\begin{array}{l}1.98 \\
1.92\end{array}$ & $\begin{array}{l}1.98 \\
1.94\end{array}$ & $\begin{array}{l}1.97 \\
1.95\end{array}$ & $\begin{array}{l}1.97 \\
1.96\end{array}$ & 0.04 (bond lengths) \\
\hline av. $r \mathrm{O}-\mathrm{Cu} / \AA$ & $\begin{array}{l}\text { DFT } \\
\text { MM }\end{array}$ & $\begin{array}{l}2.03 \\
1.99\end{array}$ & $\begin{array}{l}2.03 \\
1.99\end{array}$ & $\begin{array}{l}2.03 \\
1.99\end{array}$ & $\begin{array}{l}2.03 \\
1.99\end{array}$ & $\begin{array}{l}2.03 \\
1.99\end{array}$ & $\begin{array}{l}2.04 \\
1.99\end{array}$ & \\
\hline$\alpha /^{\circ}$ & $\begin{array}{l}\text { DFT } \\
\text { MM }\end{array}$ & $\begin{array}{l}117.3 \\
117.2\end{array}$ & $\begin{array}{l}113.4 \\
113.5\end{array}$ & $\begin{array}{l}117.8 \\
130.4\end{array}$ & $\begin{array}{l}115.0 \\
120.5\end{array}$ & $\begin{array}{l}114.0 \\
114.8\end{array}$ & $\begin{array}{l}112.6 \\
111.1\end{array}$ & 4.45 (angles) \\
\hline$\beta /{ }^{\circ}$ & $\begin{array}{l}\text { DFT } \\
\text { MM }\end{array}$ & $\begin{array}{l}92.7 \\
91.8\end{array}$ & $\begin{array}{l}91.8 \\
90.1\end{array}$ & $\begin{array}{r}91.8 \\
102.4\end{array}$ & $\begin{array}{l}92.0 \\
95.1\end{array}$ & $\begin{array}{l}91.9 \\
91.3\end{array}$ & $\begin{array}{l}91.3 \\
88.7\end{array}$ & \\
\hline$\delta /^{\circ}$ & $\begin{array}{l}\text { DFT } \\
\text { MM }\end{array}$ & $\begin{array}{l}86.4 \\
88.1\end{array}$ & $\begin{array}{l}85.8 \\
88.0\end{array}$ & $\begin{array}{l}85.7 \\
82.9\end{array}$ & $\begin{array}{l}86.0 \\
87.9\end{array}$ & $\begin{array}{l}85.8 \\
88.0\end{array}$ & $\begin{array}{l}85.2 \\
88.0\end{array}$ & \\
\hline$\varepsilon /^{\circ}$ & $\begin{array}{l}\text { DFT } \\
\text { MM }\end{array}$ & $\begin{array}{r}-10.1 \\
-6.3\end{array}$ & $\begin{array}{l}-9.1 \\
-9.1\end{array}$ & $\begin{array}{l}-8.1 \\
-9.8\end{array}$ & $\begin{array}{l}-9.7 \\
-7.9\end{array}$ & $\begin{array}{l}-9.4 \\
-9.6\end{array}$ & $\begin{array}{l}-8.8 \\
-7.3\end{array}$ & 3.24 (torsions) \\
\hline$\chi^{\prime{ }^{\circ}}$ & $\begin{array}{l}\text { DFT } \\
\text { MM }\end{array}$ & $\begin{array}{l}-8.2 \\
-6.4\end{array}$ & $\begin{array}{r}-10.8 \\
-9.1\end{array}$ & $\begin{array}{r}7.9 \\
-1.7\end{array}$ & $\begin{array}{r}-10.8 \\
-9.7\end{array}$ & $\begin{array}{r}-11.1 \\
-9.7\end{array}$ & $\begin{array}{r}-11.1 \\
-9.8\end{array}$ & \\
\hline
\end{tabular}


Table S3: Summary of Average Structural Parameters from 100 Step MM Conformational

Searches (See Table S2 for parameter definitions, standard deviations given in parentheses).

\begin{tabular}{|c|c|c|c|c|}
\hline No. & av. $\alpha /^{\circ}$ & av. $\beta /^{\circ}$ & av. $\delta /{ }^{\circ}$ & av. $\varepsilon /^{\circ}$ \\
\hline $5 \mathbf{a}$ & $117.2(0.0)$ & $91.8(0.0)$ & $88.0(0.0)$ & $-0.8(7.2)$ \\
\hline $5 b$ & $113.6(0.0)$ & $90.1(0.0)$ & $87.9(0.1)$ & $0.5(7.1)$ \\
\hline $5 c$ & $130.4(0.0)$ & $102.3(0.1)$ & $87.8(0.1)$ & $1.5(8.5)$ \\
\hline 5d & $120.5(0.0)$ & $95.1(0.0)$ & $87.8(0.1)$ & $1.0(7.4)$ \\
\hline $5 e$ & $114.9(0.0)$ & $91.2(0.0)$ & $87.9(0.1)$ & $0.3(7.1)$ \\
\hline $5 f$ & $111.4(0.2)$ & $88.9(0.2)$ & $87.9(0.1)$ & $0.4(6.9)$ \\
\hline $5 \mathrm{~h}$ & $113.7(0.2)$ & $90.5(0.2)$ & $88.3(0.5)$ & $0.7(9.0)$ \\
\hline $5 j(R)$ & $113.6(0.0)$ & $90.1(0.0)$ & $87.7(0.1)$ & $-3.0(27.3)$ \\
\hline $5 \mathrm{j}(\mathrm{S})$ & $113.6(0.0)$ & $90.1(0.0)$ & $87.8(0.1)$ & $-5.5(37.7)$ \\
\hline $6 \mathbf{b}$ & $113.6(0.0)$ & $90.4(0.1)$ & $88.0(0.1)$ & $-1.4(7.4)$ \\
\hline $6 \mathbf{i}$ & $113.6(0.0)$ & $90.1(0.0)$ & $87.9(0.1)$ & $-1.6(6.7)$ \\
\hline $7 b$ & $113.7(0.1)$ & $90.4(0.2)$ & $88.1(0.2)$ & $-0.2(6.9)$ \\
\hline $8 \mathbf{b}$ & $113.8(0.1)$ & $90.6(0.2)$ & $88.6(0.2)$ & $-5.3(8.2)$ \\
\hline $9 b$ & $114.0(0.0)$ & $91.1(0.0)$ & $89.0(0.0)$ & $-7.0(8.0)$ \\
\hline $9 \mathrm{~h}$ & $114.1(0.0)$ & $91.1(0.0)$ & $89.0(0.0)$ & $-8.9(6.2)$ \\
\hline $9 \mathrm{i}$ & $113.8(0.0)$ & $91.0(0.0)$ & $89.0(0.0)$ & $-8.9(9.7)$ \\
\hline $\mathbf{9 j}(\mathbf{R})$ & $114.0(0.1)$ & $91.0(0.0)$ & $88.5(0.2)$ & $-5.3(21.4)$ \\
\hline $9 j(S)$ & $114.0(0.1)$ & $90.5(0.2)$ & $88.6(0.2)$ & $-10.1(35.2)$ \\
\hline 10a & $117.5(0.0)$ & $92.2(0.1)$ & $88.4(0.2)$ & $-0.2(10.5)$ \\
\hline $10 b$ & $113.8(0.0)$ & $90.5(0.1)$ & $88.4(0.2)$ & $2.4(8.1)$ \\
\hline $10 \mathrm{c}$ & $130.6(0.1)$ & $102.7(0.1)$ & $88.4(0.2)$ & $-2.4(14.1)$ \\
\hline 10d & $120.8(0.0)$ & $95.5(0.1)$ & $88.4(0.2)$ & $-3.3(12.0)$ \\
\hline $10 \mathrm{e}$ & $115.1(0.0)$ & $91.6(0.1)$ & $88.4(0.2)$ & $1.5(10.1)$ \\
\hline 10f & $111.6(0.2)$ & $89.3(0.2)$ & $88.4(0.2)$ & $3.4(7.4)$ \\
\hline $10 \mathrm{~g}$ & $114.6(0.0)$ & $91.1(0.1)$ & $88.4(0.2)$ & $0.3(9.1)$ \\
\hline $10 \mathrm{~h}$ & $113.8(0.1)$ & $90.5(0.1)$ & $88.5(0.4)$ & $-1.0(12.4)$ \\
\hline$\overline{10 i} 1$ & $113.8(0.1)$ & $90.5(0.1)$ & $88.4(0.4)$ & $2.5(23.1)$ \\
\hline $10 \mathrm{j}(\mathrm{R})$ & $113.8(0.0)$ & $90.5(0.1)$ & $88.3(0.3)$ & $-0.6(26.3)$ \\
\hline $10 \mathrm{j}(\mathrm{S})$ & $113.8(0.1)$ & $90.5(0.1)$ & $88.2(0.4)$ & $-1.2(28.7)$ \\
\hline $10 \mathrm{j}(\mathbf{R})$, restr. & $113.6(0.1)$ & $90.4(0.1)$ & $88.3(0.5)$ & $-6.5(19.0)$ \\
\hline $10 \mathrm{j}(\mathrm{S})$, restr. & $113.6(0.1)$ & $90.4(0.1)$ & $88.4(0.5)$ & $-5.1(32.0)$ \\
\hline $11 \mathrm{~b}$ & $113.7(0.1)$ & $90.4(0.1)$ & $88.5(0.2)$ & $3.3(7.8)$ \\
\hline 11i & $113.7(0.1)$ & $90.4(0.1)$ & $88.4(0.2)$ & $2.4(10.3)$ \\
\hline $12 \mathrm{~b}$ & $113.8(0.2)$ & $90.7(0.3)$ & $88.6(0.2)$ & $-1.7(6.7)$ \\
\hline 13a & $117.1(0.0)$ & $91.9(0.0)$ & $88.3(0.0)$ & $-0.2(9.8)$ \\
\hline 13b & $113.6(0.0)$ & $90.3(0.0)$ & $88.2(0.0)$ & $-4.6(10.1)$ \\
\hline $13 \mathrm{c}$ & $130.5(0.0)$ & $102.6(0.0)$ & $88.4(0.0)$ & $-2.0(9.3)$ \\
\hline 13d & $120.6(0.0)$ & $95.3(0.0)$ & $88.2(0.0)$ & $1.9(7.7)$ \\
\hline $13 \mathbf{e}$ & $114.9(0.0)$ & $91.4(0.0)$ & $88.2(0.0)$ & $0.1(8.2)$ \\
\hline 13f & $111.4(0.2)$ & $89.1(0.2)$ & $88.1(0.0)$ & $1.1(8.1)$ \\
\hline $13 \mathrm{~g}$ & $114.4(0.0)$ & $90.9(0.0)$ & $88.2(0.0)$ & $0.4(9.2)$ \\
\hline $13 \mathbf{i}$ & $113.6(0.0)$ & $90.3(0.0)$ & $88.1(0.0)$ & $-3.5(14.2)$ \\
\hline $13 \mathbf{j}(\mathbf{R})$ & $113.6(0.1)$ & $90.2(0.1)$ & $87.9(0.2)$ & $-4.5(26.9)$ \\
\hline $13 \mathbf{j}(\mathbf{S})$ & $113.6(0.1)$ & $90.2(0.1)$ & $87.9(0.2)$ & $8.0(5.1)$ \\
\hline $14 a$ & $117.7(0.0)$ & $92.3(0.0)$ & $88.6(0.0)$ & $-3.3(6.9)$ \\
\hline $14 b$ & $114.0(0.0)$ & $90.7(0.1)$ & $88.6(0.1)$ & $-2.9(6.8)$ \\
\hline $14 c$ & $130.8(0.0)$ & $102.9(0.0)$ & $88.5(0.4)$ & $-2.3(7.0)$ \\
\hline 14d & $121.0(0.0)$ & $95.7(0.0)$ & $88.6(0.1)$ & $-5.5(8.7)$ \\
\hline $14 \mathrm{e}$ & $115.3(0.0)$ & $91.8(0.0)$ & $88.6(0.1)$ & $-5.9(8.5)$ \\
\hline $14 \mathrm{f}$ & $111.7(0.2)$ & $89.4(0.2)$ & $88.7(0.1)$ & $-8.0(7.2)$ \\
\hline $14 \mathrm{~g}$ & $114.8(0.0)$ & $91.3(0.0)$ & $88.6(0.1)$ & $-6.7(8.3)$ \\
\hline
\end{tabular}


Table S4: Summary of $\varepsilon$ and $\chi$ at Minima and Boltzmann Population Weighted Averages (at 213 K, 100 Step MM

Conformational Searches, see Table S2 for parameter definitions, standard deviations given in parentheses).

\begin{tabular}{|c|c|c|c|c|}
\hline No. & $\varepsilon\left(\right.$ at minimum) $/^{\circ}$ & wt. av. $\varepsilon /^{\circ}$ & $\underset{0}{\chi}($ at minimum) / & wt. av. $\chi /$ \\
\hline $5 \mathbf{a}$ & -7.8 & $-1.1(0.5)$ & -6.3 & $-0.9(0.4)$ \\
\hline $5 \mathbf{b b}$ & -7.4 & $0.2(0.3)$ & -9.1 & $0.5(0.3)$ \\
\hline $5 c$ & -9.9 & $1.2(0.2)$ & -1.7 & $-0.9(0.3)$ \\
\hline $5 d$ & -7.9 & $0.7(0.2)$ & -9.7 & $0.7(0.3)$ \\
\hline $5 e$ & -7.4 & $0.0(0.1)$ & -9.7 & $0.6(0.2)$ \\
\hline $5 f$ & -7.3 & $0.2(0.2)$ & -9.8 & $-1.0(0.2)$ \\
\hline $5 \mathrm{~h}$ & -9.4 & $0.4(0.3)$ & -8.7 & $0.3(0.3)$ \\
\hline $5 \mathbf{j}(\mathrm{R})$ & -10.7 & $-7.9(0.1)$ & -10.9 & $-1.1(0.2)$ \\
\hline $5 k(S)$ & 9.5 & $6.8(0.1)$ & 11.8 & $0.3(0.2)$ \\
\hline 6b & 8.9 & $1.0(0.6)$ & 20.0 & $19.8(1.2)$ \\
\hline $6 \mathbf{i}$ & 9.1 & $1.2(0.2)$ & 21.6 & $23.5(0.4)$ \\
\hline $7 \mathbf{b}$ & -7.1 & $1.8(0.2)$ & 34.7 & $27.7(0.8)$ \\
\hline $8 \mathbf{b}$ & -11.5 & $-9.0(0.4)$ & 39.5 & $35.1(1.2)$ \\
\hline $9 \mathrm{~b}$ & -12.0 & $-9.4(0.2)$ & 40.2 & $40.3(0.3)$ \\
\hline $9 \mathrm{~h}$ & -11.7 & $-10.3(0.3)$ & 39.4 & $39.5(0.5)$ \\
\hline $9 \mathrm{i}$ & -14.8 & $-11.7(0.2)$ & 39.8 & $40.0(0.3)$ \\
\hline 9j (R) & -25.6 & $-10.6(0.3)$ & 41.0 & $40.5(0.3)$ \\
\hline 9k (S) & -3.7 & $-1.0(0.1)$ & 40.9 & $40.2(0.4)$ \\
\hline 10a & 12.7 & $7.3(0.3)$ & 22.2 & $25.0(0.5)$ \\
\hline $10 b$ & 12.2 & $6.1(0.1)$ & 23.2 & $27.1(0.3)$ \\
\hline 10c & $9.7 / 8.4^{\mathrm{a}}$ & $5.2(0.1)$ & $41.4 / 34.8^{\mathrm{a}}$ & $31.3(0.4)$ \\
\hline 10d & 12.1 & $5.9(0.2)$ & 25.7 & $28.4(0.4)$ \\
\hline 10e & 12.0 & $7.7(0.1)$ & 24.2 & $26.9(0.3)$ \\
\hline $10 f$ & 11.9 & $-4.4(0.2)$ & 23.6 & $26.9(0.5)$ \\
\hline $10 \mathrm{~g}$ & 12.3 & $5.9(0.1)$ & 23.3 & $26.4(0.3)$ \\
\hline 10h & 5.5 & $2.4(0.1)$ & 43.8 & $43.4(0.7)$ \\
\hline 10i_1 & -6.1 & $0.6(0.2)$ & 42.4 & $42.1(1.0)$ \\
\hline $10 \mathrm{j}(\mathrm{R})$ & 5.7 & $2.4(0.1)$ & 25.1 & $26.5(0.4)$ \\
\hline $10 \mathrm{k}(\mathrm{S})$ & 20.0 & $16.4(0.4)$ & 7.0 & $16.8(0.2)$ \\
\hline $10 \mathrm{j}(\mathrm{R})$, restr. & -23.8 & $-7.5(0.6)$ & 48.2 & $42.6(1.7)$ \\
\hline $10 \mathrm{k}(\mathrm{S})$, restr. & -1.6 & $-0.1(0.1)$ & 48.4 & $47.5(2.0)$ \\
\hline $11 b$ & -8.9 & $0.1(0.3)$ & 37.5 & $32.5(1.0)$ \\
\hline $11 \mathrm{i}$ & -11.3 & $-4.6(0.4)$ & 37.1 & $32.8(1.3)$ \\
\hline $12 \mathrm{~b}$ & -8.3 & $-2.7(0.4)$ & 34.0 & $33.0(1.8)$ \\
\hline $13 \mathbf{a}$ & 8.3 & $0.2(2.5)$ & 19.5 & $19.5(0.3)$ \\
\hline $13 b$ & -11.1 & $-6.6(1.6)$ & 20.5 & $20.5(0.8)$ \\
\hline $13 c$ & -11.1 & $-3.9(0.7)$ & 19.1 & $25.3(0.6)$ \\
\hline 13d & 9.1 & $2.4(1.0)$ & 22.3 & $22.7(0.1)$ \\
\hline $13 \mathrm{e}$ & 8.4 & $0.5(0.5)$ & 21.2 & $21.0(0.2)$ \\
\hline 13f & 8.1 & $3.7(0.5)$ & 20.4 & $20.2(1.0)$ \\
\hline $13 g$ & 7.7 & $1.5(1.3)$ & 20.6 & $20.5(0.4)$ \\
\hline $13 \mathbf{i}$ & -16.8 & $-6.7(1.9)$ & 18.8 & $19.1(0.6)$ \\
\hline 13j (R) & -26.0 & $-17.0(0.9)$ & 19.5 & $18.7(0.6)$ \\
\hline 13k (S) & 11.6 & $10.0(0.6)$ & 11.2 & $14.6(0.3)$ \\
\hline $14 a$ & -9.1 & $-2.8(1.0)$ & 33.3 & $33.3(4.1)$ \\
\hline $14 b$ & -8.8 & $-3.5(0.6)$ & 35.6 & $35.4(2.6)$ \\
\hline $14 c$ & -7.7 & $-1.0(0.3)$ & 36.3 & $40.3(1.6)$ \\
\hline 14d & -11.2 & $-7.8(1.0)$ & 36.3 & $36.3(3.1)$ \\
\hline $14 e$ & -11.3 & $-9.3(0.6)$ & 35.9 & $35.6(1.8)$ \\
\hline $14 f$ & -11.2 & $-11.2(0.7)$ & 34.6 & $35.5(2.4)$ \\
\hline $14 g$ & -11.2 & $-9.2(1.1)$ & 34.9 & $34.9(3.5)$ \\
\hline
\end{tabular}

${ }^{a}$ energetically equivalent conformations 
Table S5: Diastereomeric Excess (de) Calculated from MM Transition State Conformational Searches (Refer to main paper for reference numbers).

\begin{tabular}{|c|c|c|c|c|c|c|c|c|c|}
\hline No. & $\mathbf{T} / \mathbf{K}$ & $\begin{array}{l}\text { calc. de } \\
/ \%\end{array}$ & $\begin{array}{l}\text { expt. de } \\
/ \%\end{array}$ & Ref. & No. & $\mathbf{T} / \mathbf{K}$ & $\begin{array}{l}\text { calc. de } \\
/ \%\end{array}$ & $\begin{array}{l}\text { expt. de } \\
/ \%\end{array}$ & Ref. \\
\hline $5 \mathbf{5 a}$ & 213 & 91.0 & & & 11b & 298 & 70.4 & & \\
\hline $5 b$ & 213 & 91.0 & & & $11 \mathrm{i}$ & 298 & 88.9 & 82 & 63 \\
\hline $5 \mathrm{5h}$ & 213 & 94.3 & & & $12 b$ & 213 & 91.7 & & \\
\hline $6 \mathbf{b}$ & 213 & 91.2 & & & 13a & 195 & 94.7 & 98 & 7 \\
\hline $6 \mathbf{i}$ & 213 & 92.5 & & & 13b & 223 & 91.0 & 96 & 13 \\
\hline $7 b$ & 213 & 88.6 & & & $13 c$ & 223 & 88.0 & 96 & 13 \\
\hline $8 b$ & 195 & 94.3 & 92 & 10 & 13d & 223 & 91.6 & 94 & 13 \\
\hline $9 \mathrm{~b}$ & 195 & 92.5 & 96 & 10 & $13 e$ & 223 & 91.8 & 94 & 13 \\
\hline $9 \mathrm{~h}$ & 213 & 92.3 & & & 13f & 223 & 90.2 & 92 & 13 \\
\hline$\overline{9 i}$ & 258 & 88.0 & 92 & 10 & $13 g$ & 223 & 90.7 & 95 & 14 \\
\hline $10 a$ & 213 & 89.6 & & & $13 i$ & 213 & 95.4 & & \\
\hline $10 \mathrm{~b}$ & 195 & 92.6 & 90 & 10 & $14 a$ & 213 & 92.7 & & \\
\hline 10c & 213 & 82.3 & & & 14b & 213 & 90.1 & & \\
\hline 10d & 213 & 87.7 & & & $14 c$ & 213 & 89.1 & & \\
\hline $10 \mathrm{e}$ & 213 & 90.4 & & & 14d & 213 & 88.8 & & \\
\hline $10 f$ & 223 & 92.7 & & & $14 \mathrm{e}$ & 213 & 86.7 & & \\
\hline $10 \mathrm{~g}$ & 223 & 87.9 & & 14 & $14 f$ & 213 & 96.9 & & \\
\hline $10 \mathrm{~h}$ & 298 & 56.4 & & & $14 g$ & 223 & 90.4 & 92 & 14 \\
\hline 10i_1 & 298 & 63.8 & 23 & 63 & & & & & \\
\hline
\end{tabular}


Table S6: Boltzmann Population Weighted Averages of $\varepsilon$ and $\chi$ for 1000 Step MM Transition State Conformational Searches (at $213 \mathrm{~K}$, see Table S2 for parameter definitions, excluding all type j complexes).

\begin{tabular}{|c|c|c|c|c|c|c|c|c|}
\hline & \multicolumn{4}{|c|}{$\varepsilon /^{\circ}$} & \multicolumn{4}{|c|}{$x^{1^{\circ}}$} \\
\hline No. & $n 1$ & $n 2$ & $x 1$ & $x 2$ & $n 1$ & $n 2$ & $x 1$ & $x 2$ \\
\hline $5 a$ & 4 & -6 & 8 & -8 & 1 & -1 & 0 & 0 \\
\hline $5 b$ & 6 & & 8 & -5 & 0 & -1 & 0 & 0 \\
\hline $5 \mathrm{Sh}$ & 6 & & 10 & -10 & 2 & 2 & 0 & $\overline{0}$ \\
\hline $6 b$ & 0 & -9 & 7 & -11 & 19 & 33 & 19 & 30 \\
\hline $6 \mathbf{i}$ & -2 & -8 & 7 & -10 & 22 & 34 & 23 & 32 \\
\hline $7 b$ & -2 & -9 & 9 & -10 & 31 & 40 & 32 & 39 \\
\hline $8 b$ & 0 & -8 & 8 & -11 & 38 & 49 & 38 & 39 \\
\hline $9 \mathrm{~b}$ & -1 & -6 & 8 & -8 & 40 & 49 & 43 & 47 \\
\hline $9 \mathrm{~h}$ & -1 & -6 & 8 & -9 & 39 & 47 & 41 & 43 \\
\hline $9 \mathbf{i}$ & 4 & -8 & 10 & -10 & 38 & 50 & 41 & 50 \\
\hline $10 \mathrm{a}$ & 3 & -10 & 10 & -15 & 37 & 38 & 42 & 22 \\
\hline $10 \mathrm{~b}$ & 4 & 8 & 10 & 15 & 37 & 30 & 43 & 24 \\
\hline $10 \mathrm{c}$ & 5 & -7 & 11 & -14 & 34 & 28 & 39 & 36 \\
\hline 10d & 3 & -9 & 10 & -15 & 39 & 39 & 45 & 28 \\
\hline $10 \mathrm{e}$ & 4 & -8 & 10 & -15 & 37 & 29 & 44 & 22 \\
\hline $10 f$ & 4 & -8 & 10 & -15 & 37 & 40 & 43 & 21 \\
\hline $10 \mathrm{~g}$ & 5 & -9 & 10 & -15 & 36 & 43 & 42 & 23 \\
\hline $10 \mathrm{~h}$ & 2 & -3 & 10 & -9 & 39 & 27 & 42 & 36 \\
\hline 10i_1 & 5 & 12 & 12 & -11 & 37 & 45 & 41 & 43 \\
\hline $11 b$ & 2 & -7 & 8 & -9 & 36 & 31 & 30 & 37 \\
\hline $11 i$ & 4 & -5 & 9 & -7 & 36 & 34 & 33 & 37 \\
\hline $12 \mathrm{~b}$ & 4 & -8 & 8 & -7 & 53 & 43 & 47 & 40 \\
\hline $13 \mathbf{a}$ & 0 & -8 & 8 & -9 & 23 & 32 & 24 & 32 \\
\hline $13 b$ & 0 & -9 & 9 & -10 & 25 & 33 & 27 & 33 \\
\hline $13 c$ & 2 & -11 & 9 & -10 & 26 & 36 & 30 & 36 \\
\hline 13d & -1 & -9 & 8 & -10 & 26 & 34 & 28 & 35 \\
\hline $13 \mathrm{e}$ & -1 & -9 & 8 & -10 & 24 & 33 & 26 & 34 \\
\hline 13f & 1 & -9 & 9 & -9 & 24 & 33 & 26 & 33 \\
\hline $13 \mathrm{~g}$ & 1 & -8 & 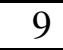 & -9 & 24 & 33 & 26 & 33 \\
\hline $13 i$ & 4 & -9 & 10 & -11 & 22 & 36 & 25 & 33 \\
\hline $14 a$ & 1 & -7 & 7 & -10 & 34 & 45 & 42 & 45 \\
\hline $14 \mathrm{~b}$ & 2 & -7 & 8 & -10 & 35 & 42 & 43 & 44 \\
\hline $14 \mathrm{c}$ & 1 & -8 & 7 & -10 & 34 & 39 & 42 & 49 \\
\hline 14d & -1 & -8 & 8 & -10 & 36 & 41 & 44 & 45 \\
\hline $14 \mathrm{e}$ & 2 & -8 & 8 & -10 & 36 & 40 & 43 & 46 \\
\hline $14 f$ & 2 & -7 & 2 & -10 & 36 & 48 & 47 & 46 \\
\hline $14 \mathrm{~g}$ & 1 & -7 & 9 & -10 & 36 & 49 & 43 & 48 \\
\hline
\end{tabular}


Table S7: Summary of Extended Validation Transition State Searches for $\mathbf{9 b}$ and $\mathbf{1 3 b}$.

\begin{tabular}{|c|c|c|c|c|c|c|}
\hline No. & $\begin{array}{l}\mathbf{r}(\alpha-\mathrm{C}- \\
\mathrm{C}) \\
/ \AA\end{array}$ & $\begin{array}{l}\mathbf{r}(\beta-\mathrm{C}-\mathrm{C}) \\
/ \AA\end{array}$ & $\begin{array}{l}\mathbf{T} \\
/ \mathbf{K}\end{array}$ & $\begin{array}{l}\text { de } \\
/ \%\end{array}$ & $\begin{array}{l}\text { ee } \\
1 \%\end{array}$ & Ref. \\
\hline $9 \mathrm{~b}$ & 1.70 & 2.85 & 195 & 92.5 & 75.6 & \\
\hline $9 \mathrm{~b}$ & 1.80 & 2.85 & 195 & 90.3 & 70.0 & \\
\hline $9 \mathrm{~b}$ & 1.90 & 2.85 & 195 & 89.9 & 90.8 & \\
\hline $9 \mathrm{~b}$ & 1.90 & 2.95 & 195 & 88.3 & 82.7 & \\
\hline 9b & \multicolumn{2}{|c|}{ expt. } & 195 & 96 & 98 & 10 \\
\hline 13b & 1.70 & 2.85 & 223 & 91.0 & 26.3 & \\
\hline 13b & 2.00 & 2.75 & 223 & 87.4 & 26.7 & \\
\hline $13 b$ & 1.90 & 2.85 & 223 & 88.8 & 29.3 & \\
\hline 13b & 2.00 & 2.95 & 223 & 88.3 & 10.4 & \\
\hline 13b & & & 223 & 98 & 98 & 13 \\
\hline
\end{tabular}


Figure S1: $\mathrm{O}=\mathrm{C}-\mathrm{C}=\mathrm{C}(\varepsilon)$ Rotational Energy Profiles for Catalyst-Substrate Complexes of 5b, 9b, $13 b$ and $14 b$.

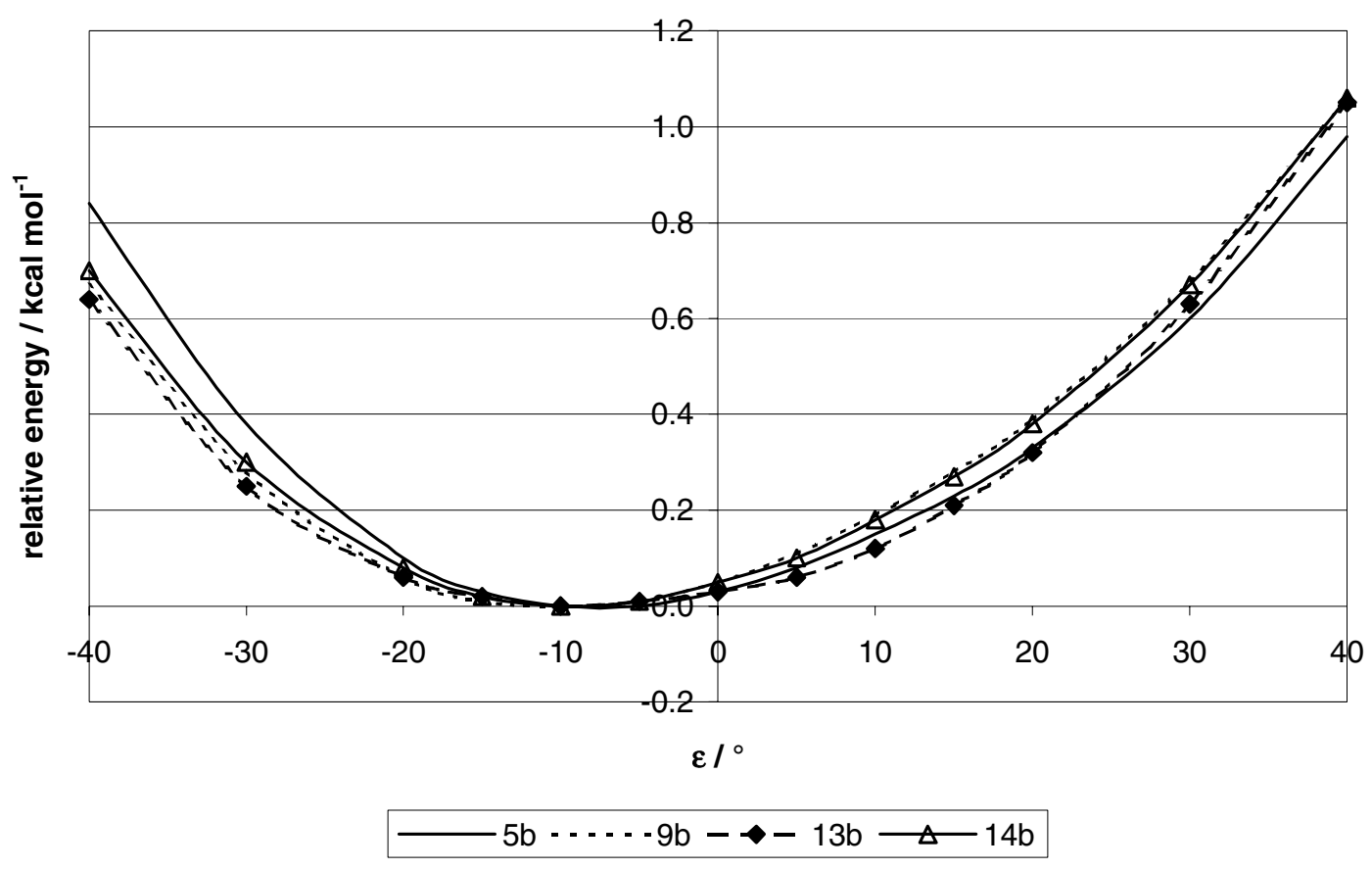


Figure S2: DFT Calculated Transition State Geometries $(n 1, n 2)$ for $\mathbf{5 b}$.
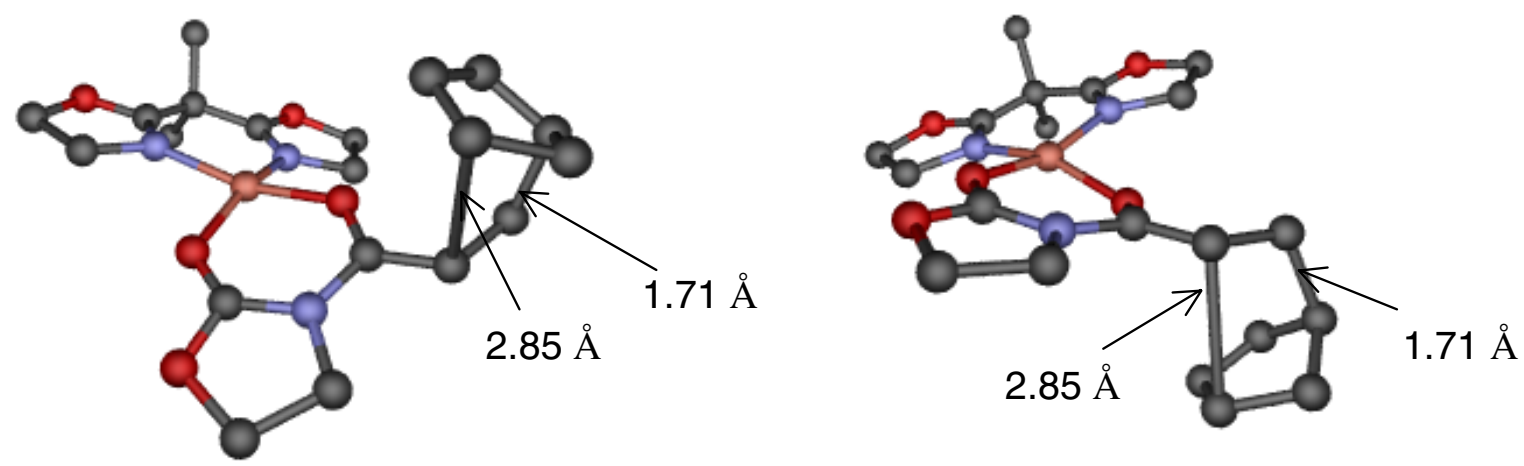
Figure S3: Ligand Plane Twist Angle $\chi$ Rotational Energy Profiles for Transition States of 9b.

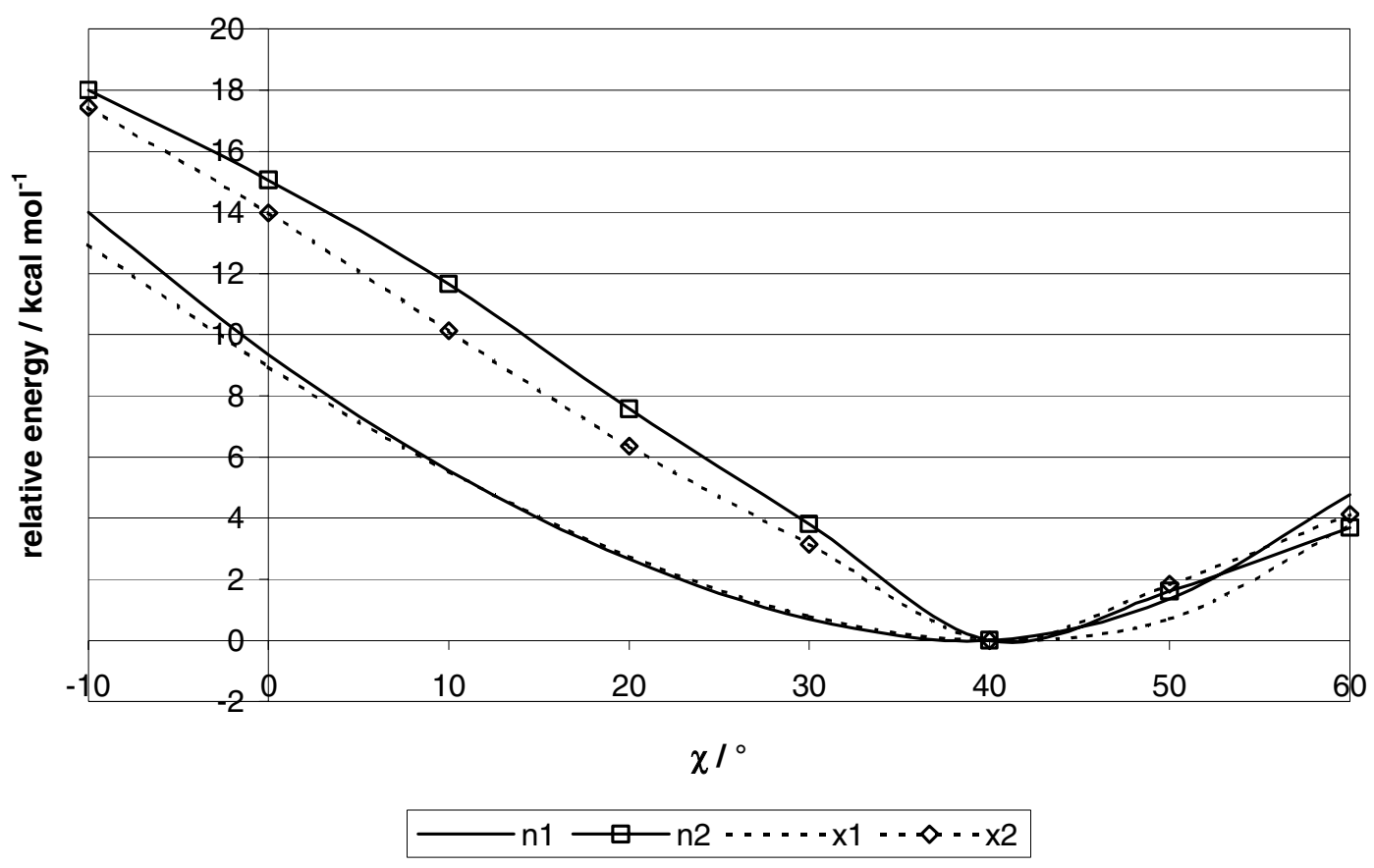




\section{DFT Calculated Structural Coordinates}

$3\left(\mathrm{R}, \mathrm{R}^{\prime}, \mathrm{R}^{\prime \prime}=\mathrm{H}\right)$

$\begin{array}{rlrrr}1 & \mathrm{C} & 1.0190 & 1.2130 & -0.6290 \\ 2 & \mathrm{C} & -0.2700 & 1.9250 & -0.3390 \\ 3 & \mathrm{H} & -0.1870 & 2.4130 & 0.6510 \\ 4 & \mathrm{H} & -0.3860 & 2.7530 & -1.0580 \\ 5 & \mathrm{C} & -1.5170 & 1.0910 & -0.3550 \\ 6 & \mathrm{~N} & -1.6140 & -0.2050 & -0.3400 \\ 7 & \mathrm{Cu} & -0.0990 & -1.5090 & -0.3710 \\ 8 & \mathrm{~N} & 1.2400 & -0.0690 & -0.6730 \\ 9 & \mathrm{O} & -1.4190 & -3.0230 & -0.2710 \\ 10 & \mathrm{C} & -1.1820 & -4.2400 & -0.1470 \\ 11 & \mathrm{~N} & 0.0660 & -4.8600 & -0.0160 \\ 12 & \mathrm{C} & 1.2860 & -4.1800 & -0.0200 \\ 13 & \mathrm{O} & 1.3510 & -2.9280 & -0.1890 \\ 14 & \mathrm{O} & -2.6490 & 1.7910 & -0.3660 \\ 15 & \mathrm{C} & -3.7780 & 0.8050 & -0.4260 \\ 16 & \mathrm{H} & -4.4490 & 1.0420 & 0.4070 \\ 17 & \mathrm{H} & -4.2800 & 0.9700 & -1.3870 \\ 18 & \mathrm{C} & -3.0730 & -0.5690 & -0.3080 \\ 19 & \mathrm{H} & -3.2950 & -1.0820 & 0.6370 \\ 20 & \mathrm{H} & -3.3110 & -1.2420 & -1.1400 \\ 21 & \mathrm{C} & 2.7020 & -0.2820 & -0.9430 \\ 22 & \mathrm{H} & 2.8290 & -0.9300 & -1.8200 \\ 23 & \mathrm{H} & 3.1610 & -0.7630 & -0.0700 \\ 24 & \mathrm{C} & 3.2290 & 1.1540 & -1.1810 \\ 25 & \mathrm{H} & 3.4890 & 1.3670 & -2.2250 \\ 26 & \mathrm{H} & 4.0410 & 1.4560 & -0.5110 \\ 27 & \mathrm{O} & 2.0530 & 2.0200 & -0.8540 \\ 28 & \mathrm{O} & -2.1700 & -5.1210 & -0.1210 \\ 29 & \mathrm{C} & -1.6380 & -6.5070 & -0.0640 \\ 30 & \mathrm{H} & -1.8790 & -6.9750 & -1.0270 \\ 31 & \mathrm{H} & -2.1610 & -7.0150 & 0.7550 \\ 32 & \mathrm{C} & -0.1200 & -6.3370 & 0.1770 \\ 33 & \mathrm{H} & 0.1730 & -6.6180 & 1.1980 \\ 34 & \mathrm{H} & 0.4710 & -6.8990 & -0.5550 \\ 35 & \mathrm{C} & 2.4950 & -4.9870 & 0.1890 \\ 36 & \mathrm{H} & 2.3750 & -6.0520 & 0.3810 \\ 37 & \mathrm{C} & 3.7320 & -4.4480 & 0.1560 \\ 38 & \mathrm{H} & 4.6170 & -5.0690 & 0.3150 \\ 39 & \mathrm{H} & 3.8890 & -3.3850 & -0.0340 \\ & & & & \end{array}$

$5 a$

$\begin{array}{rlrrr}1 & \mathrm{C} & -2.2256 & -2.7085 & 0.4065 \\ 2 & \mathrm{C} & -1.5863 & -3.3413 & -0.7937 \\ 3 & \mathrm{H} & -1.3767 & -4.4022 & -0.5664 \\ 4 & \mathrm{H} & -2.3299 & -3.3700 & -1.6112 \\ 5 & \mathrm{C} & -0.3286 & -2.7108 & -1.3137 \\ 6 & \mathrm{~N} & 0.2990 & -1.6635 & -0.8649 \\ 7 & \mathrm{Cu} & -0.2449 & -0.5285 & 0.6756 \\ 8 & \mathrm{~N} & -1.8470 & -1.6483 & 1.0571 \\ 9 & \mathrm{O} & -0.8116 & 0.7939 & 2.0867 \\ 10 & \mathrm{C} & -0.1603 & 1.7524 & 2.5411 \\ 11 & \mathrm{~N} & 1.0944 & 2.2114 & 2.1154 \\ 12 & \mathrm{C} & 1.8404 & 1.6195 & 1.0963 \\ 13 & \mathrm{O} & 1.4548 & 0.5616 & 0.5196 \\ 14 & \mathrm{O} & 0.1970 & -3.3279 & -2.3704 \\ 15 & \mathrm{C} & 1.3740 & -2.5162 & -2.8146 \\ 16 & \mathrm{H} & 2.2124 & -3.2109 & -2.9354 \\ 17 & \mathrm{H} & 1.0970 & -2.0716 & -3.7790 \\ 18 & \mathrm{C} & 1.5491 & -1.4850 & -1.6777 \\ 19 & \mathrm{H} & 2.4192 & -1.6978 & -1.0410 \\ 20 & \mathrm{H} & 1.6161 & -0.4553 & -2.0474 \\ 21 & \mathrm{C} & -2.8673 & -1.3651 & 2.1258 \\ 22 & \mathrm{H} & -2.3747 & -1.2315 & 3.0957 \\ 23 & \mathrm{H} & -3.4044 & -0.4413 & 1.8704 \\ 24 & \mathrm{C} & -3.7686 & -2.6192 & 2.0806 \\ 25 & \mathrm{H} & -3.5976 & -3.3200 & 2.9076 \\ 26 & \mathrm{H} & -4.8375 & -2.4099 & 1.9645 \\ 27 & \mathrm{O} & -3.3262 & -3.3264 & 0.8327 \\ 28 & \mathrm{O} & -0.6314 & 2.4863 & 3.5379 \\ 29 & \mathrm{C} & 0.3905 & 3.4705 & 3.9853 \\ 30 & \mathrm{H} & 0.7633 & 3.1139 & 4.9549 \\ 31 & \mathrm{H} & -0.1200 & 4.4343 & 4.0966 \\ 32 & \mathrm{C} & 1.4591 & 3.4535 & 2.8726 \\ 33 & \mathrm{H} & 2.4723 & 3.3752 & 3.2839 \\ 34 & \mathrm{H} & 1.3842 & 4.3265 & 2.2095 \\ 35 & \mathrm{C} & 3.0973 & 2.2808 & 0.7223 \\ 36 & \mathrm{H} & 3.2995 & 3.2692 & 1.1346 \\ 37 & \mathrm{C} & 4.0008 & 1.7032 & -0.1021 \\ 38 & \mathrm{H} & 4.9281 & 2.2200 & -0.3628 \\ 39 & \mathrm{H} & 3.8439 & 0.7070 & -0.5199\end{array}$




$\begin{array}{rlrrr}1 & \mathrm{C} & 1.0158 & 2.1831 & 1.8046 \\ 2 & \mathrm{C} & 1.2318 & 1.4353 & 3.1101 \\ 3 & \mathrm{C} & 0.5907 & 2.2746 & 4.2708 \\ 4 & \mathrm{H} & -0.4955 & 2.3869 & 4.1343 \\ 5 & \mathrm{H} & 0.7793 & 1.7733 & 5.2281 \\ 6 & \mathrm{H} & 1.0500 & 3.2705 & 4.2977 \\ 7 & \mathrm{C} & 2.7751 & 1.2878 & 3.3506 \\ 8 & \mathrm{H} & 2.9497 & 0.7755 & 4.3047 \\ 9 & \mathrm{H} & 3.2311 & 2.2843 & 3.3969 \\ 10 & \mathrm{H} & 3.2554 & 0.7133 & 2.5441 \\ 11 & \mathrm{C} & 0.6110 & 0.0483 & 3.1222 \\ 12 & \mathrm{~N} & -0.0582 & -0.5779 & 2.1955 \\ 13 & \mathrm{Cu} & -0.5389 & 0.0943 & 0.3998 \\ 14 & \mathrm{~N} & 0.3751 & 1.8180 & 0.7305 \\ 15 & \mathrm{O} & -1.2956 & 0.7798 & -1.3464 \\ 16 & \mathrm{C} & -1.8762 & 0.1365 & -2.2385 \\ 17 & \mathrm{~N} & -2.2328 & -1.2198 & -2.2211 \\ 18 & \mathrm{C} & -1.9550 & -2.0868 & -1.1630 \\ 19 & \mathrm{O} & -1.2897 & -1.7102 & -0.1569 \\ 20 & \mathrm{O} & 0.7938 & -0.6192 & 4.2664 \\ 21 & \mathrm{C} & 0.0284 & -1.8973 & 4.1721 \\ 22 & \mathrm{H} & -0.8249 & -1.8012 & 4.8563 \\ 23 & \mathrm{H} & 0.6990 & -2.6987 & 4.5023 \\ 24 & \mathrm{C} & -0.3717 & -1.9624 & 2.6851 \\ 25 & \mathrm{H} & 0.2283 & -2.6847 & 2.1137 \\ 26 & \mathrm{H} & -1.4353 & -2.1852 & 2.5423 \\ 27 & \mathrm{C} & 0.3721 & 2.9679 & -0.2396 \\ 28 & \mathrm{H} & 0.6839 & 2.6301 & -1.2342 \\ 29 & \mathrm{H} & -0.6464 & 3.3751 & -0.3068 \\ 30 & \mathrm{C} & 1.3735 & 3.9498 & 0.3992 \\ 31 & \mathrm{H} & 2.3622 & 3.9463 & -0.0782 \\ 32 & \mathrm{H} & 1.0024 & 4.9744 & 0.5114 \\ 33 & \mathrm{O} & 1.5680 & 3.4009 & 1.7771 \\ 34 & \mathrm{O} & -2.2464 & 0.7190 & -3.3708 \\ 35 & \mathrm{C} & -2.7846 & -0.2893 & -4.3210 \\ 36 & \mathrm{H} & -3.7077 & 0.1262 & -4.7423 \\ 37 & \mathrm{H} & -2.0247 & -0.4189 & -5.1037 \\ 38 & \mathrm{C} & -3.0031 & -1.5549 & -3.4625 \\ 39 & \mathrm{H} & -2.5925 & -2.4496 & -3.9472 \\ 40 & \mathrm{H} & -4.0627 & -1.7105 & -3.2121 \\ 41 & \mathrm{C} & -2.4717 & -3.4581 & -1.2730 \\ 42 & \mathrm{H} & -3.1584 & -3.6847 & -2.0887 \\ & \mathrm{H} & -2.1192 & -4.4343 & -0.4052 \\ & & -2.5198 & -5.4459 & -0.5105\end{array}$

$\begin{array}{rlrrr}1 & \mathrm{Cu} & -0.0469 & -0.0983 & -0.0270 \\ 2 & \mathrm{~N} & 1.9436 & -0.0801 & 0.0280 \\ 3 & \mathrm{~N} & -0.1336 & 1.8986 & 0.0171 \\ 4 & \mathrm{C} & 2.7029 & 0.9698 & 0.1126 \\ 5 & \mathrm{C} & 0.8711 & 2.7180 & 0.0601 \\ 6 & \mathrm{C} & 2.3653 & 2.4526 & 0.1442 \\ 7 & \mathrm{O} & 4.0647 & 0.7296 & 0.1729 \\ 8 & \mathrm{O} & 0.5612 & 4.0633 & 0.0392 \\ 9 & \mathrm{C} & 4.2608 & -0.7964 & 0.1184 \\ 10 & \mathrm{C} & -0.9725 & 4.1734 & -0.0853 \\ 11 & \mathrm{C} & 2.7979 & -1.3297 & 0.0147 \\ 12 & \mathrm{C} & -1.4303 & 2.6828 & -0.0577 \\ 13 & \mathrm{C} & 2.9063 & 3.0665 & 1.4918 \\ 14 & \mathrm{C} & 3.0697 & 3.1490 & -1.0828 \\ 15 & \mathrm{H} & 2.6131 & -1.8707 & -0.9207 \\ 16 & \mathrm{H} & -2.0314 & 2.4456 & 0.8279 \\ 17 & \mathrm{H} & -1.1561 & 4.6872 & -1.0324 \\ 18 & \mathrm{H} & -1.2992 & 4.7669 & 0.7719 \\ 19 & \mathrm{H} & -1.9727 & 2.3904 & -0.9620 \\ 20 & \mathrm{H} & 4.7808 & -1.0626 & 1.0420 \\ 21 & \mathrm{H} & 4.8761 & -0.9858 & -0.7647 \\ 22 & \mathrm{H} & 2.5164 & -1.9538 & 0.8681 \\ 23 & \mathrm{H} & 2.4248 & 2.5928 & 2.3600 \\ 24 & \mathrm{H} & 3.9900 & 2.9088 & 1.5480 \\ 25 & \mathrm{H} & 2.6926 & 4.1420 & 1.5047 \\ 26 & \mathrm{H} & 2.7019 & 2.7343 & -2.0327 \\ 27 & \mathrm{H} & 2.8556 & 4.2238 & -1.0532 \\ 28 & \mathrm{H} & 4.1520 & 2.9889 & -1.0137 \\ 29 & \mathrm{O} & -0.2404 & -2.0062 & 0.5604 \\ 30 & \mathrm{O} & -1.9502 & -0.1587 & -0.6933 \\ 31 & \mathrm{C} & -1.1222 & -2.9008 & 0.2206 \\ 32 & \mathrm{C} & -2.5809 & -1.2139 & -0.9265 \\ 33 & \mathrm{~N} & -2.2751 & -2.5181 & -0.5591 \\ 34 & \mathrm{O} & -3.7679 & -1.1699 & -1.6368 \\ 35 & \mathrm{C} & -4.2916 & -2.5909 & -1.8624 \\ 36 & \mathrm{C} & -3.3431 & -3.4885 & -1.0060 \\ 37 & \mathrm{C} & -1.0228 & -4.2491 & 0.6178 \\ 38 & \mathrm{C} & 0.1404 & -4.7821 & 1.3447 \\ 39 & \mathrm{H} & -5.3295 & -2.5869 & -1.5239 \\ 40 & \mathrm{H} & -4.2136 & -2.7705 & -2.9375 \\ 41 & \mathrm{H} & -3.8565 & -3.8953 & -0.1266 \\ 42 & \mathrm{H} & -2.8969 & -4.2890 & -1.6056 \\ 43 & \mathrm{H} & -1.7907 & -4.9468 & 0.3031 \\ 44 & \mathrm{H} & 1.0481 & -4.1870 & 1.2091 \\ 45 & \mathrm{H} & 0.3367 & -5.8284 & 1.0766 \\ 46 & \mathrm{C} & -0.1306 & -4.8698 & 3.0304 \\ 47 & \mathrm{C} & -2.3058 & -3.9421 & 3.1310 \\ 48 & \mathrm{C} & -0.1047 & -3.4922 & 3.5475 \\ 49 & \mathrm{C} & -1.3941 & -2.9392 & 3.5051 \\ 50 & \mathrm{C} & -1.6242 & -5.2834 & 3.1951 \\ 51 & \mathrm{H} & 0.6248 & -5.5525 & 3.4320 \\ 52 & \mathrm{H} & 0.7996 & -2.9618 & 3.8427 \\ 53 & \mathrm{H} & -1.6462 & -1.9045 & 3.7232 \\ 56 & \mathrm{H} & -3.3783 & -3.7971 & 3.0051 \\ & \mathrm{H} & -1.9979 & -5.6783 & 4.2257 \\ & & -6.0424 & 2.4991\end{array}$


$5 \mathrm{~b}, n 2$

\begin{tabular}{|c|c|c|c|c|}
\hline & $\mathrm{Cu}$ & 0.0468 & .0982 & \\
\hline 2 & $\mathrm{~N}$ & -1.9437 & 0.0800 & 0280 \\
\hline 3 & $\mathrm{~N}$ & 0.1336 & -1.8987 & 0.0171 \\
\hline & $\mathrm{C}$ & -2.7031 & -0.9699 & \\
\hline 5 & $\mathrm{C}$ & -0.8711 & -2.7181 & 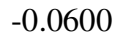 \\
\hline 6 & $\mathrm{C}$ & -2.3653 & -2.4527 & -0.1444 \\
\hline & $\mathrm{O}$ & -4.0649 & -0.7296 & - 172 \\
\hline 8 & $\mathrm{O}$ & -0.5611 & -4.0636 & -0.039 \\
\hline 9 & $\mathrm{C}$ & -4.2608 & 0.7965 & -0.1 \\
\hline & $\mathrm{C}$ & 0.9727 & -4.1735 & \\
\hline & $\mathrm{C}$ & -2.7979 & & -0.014 \\
\hline & $\mathrm{C}$ & 1.4303 & -2.6829 & \\
\hline & $\mathrm{C}$ & -2.9064 & -3.00 & \\
\hline & $\mathrm{C}$ & -3.0696 & -3.14 & \\
\hline & $\mathrm{O}$ & -2.6131 & 1.8707 & 0.920 \\
\hline & $\mathrm{O}$ & & & \\
\hline & $\mathrm{C}$ & & -4.6 & \\
\hline & $\mathrm{C}$ & 1.29 & -4.7 & -0.772 \\
\hline & $\mathrm{N}$ & & & \\
\hline & $\mathrm{O}$ & -4.7809 & 1.00 & -1.0420 \\
\hline & $\mathrm{C}$ & -4.8 & 0.9 & \\
\hline & $\mathrm{C}$ & -2.5 & & \\
\hline & $\mathrm{C}$ & -2.4250 & -2.5930 & -2.3598 \\
\hline & $\mathrm{C}$ & -3.9 & -2.90 & \\
\hline & $\mathrm{H}$ & -2.6 & -4.1 & \\
\hline & $\mathrm{H}$ & -2.7017 & -2.73 & 2.0325 \\
\hline & $\mathrm{H}$ & -2.8 & -4.2 & \\
\hline & $\mathrm{H}$ & -4.1 & -2. & \\
\hline & $\mathrm{H}$ & 0.24 & 2.0061 & -0.5604 \\
\hline & $\mathrm{H}$ & 1.9 & & \\
\hline & $\mathrm{H}$ & 1.1 & & -0.220 \\
\hline & $\mathrm{H}$ & 2.5 & 1.2 & 0.926 \\
\hline & $\mathrm{H}$ & $2.2^{7}$ & & \\
\hline & $\mathrm{H}$ & & & $1.636^{\prime}$ \\
\hline & $\mathrm{H}$ & 4.29 & 2.55 & 1.862 \\
\hline & $\mathrm{H}$ & 3.3 & 3.4 & 1.005 \\
\hline & $\mathrm{H}$ & 1.02 & 4.2 & -0.6179 \\
\hline & $\mathrm{H}$ & -0.14 & 4.78 & -1.344 \\
\hline 9 & $\mathrm{H}$ & 5.32 & 2.58 & 1.523 \\
\hline & $\mathrm{H}$ & 4.2 & 2.7 & 2.937 \\
\hline & $\mathrm{H}$ & 3.85 & 3.8 & \\
\hline & $\mathrm{H}$ & 2.89 & & 1.605 \\
\hline & $\mathrm{H}$ & 1.7905 & 4.9 & -0.303 \\
\hline & $\mathrm{H}$ & -1.0481 & 4.18 & -1.209 \\
\hline & $\mathrm{H}$ & -0.33 & & -1.076 \\
\hline & $\mathrm{C}$ & & & $-3.030 t$ \\
\hline & $\mathrm{C}$ & 2.3059 & 3.9420 & -3.130 \\
\hline & $\mathrm{C}$ & 0.1049 & 3.4928 & $-3.547^{\prime}$ \\
\hline & $\mathrm{C}$ & & 2.93 & -3.504 \\
\hline 50 & $\mathrm{C}$ & 1.6247 & 5.2835 & -3.195 \\
\hline & $\mathrm{H}$ & -0.6245 & & -3.432 \\
\hline & $\mathrm{H}$ & -0.7994 & & \\
\hline & $\mathrm{H}$ & 1.6462 & 1.9047 & -3.723 \\
\hline & $\mathrm{H}$ & 3.3785 & 3.7971 & -3.004 \\
\hline & $\mathrm{H}$ & & 5.6784 & -4.225 \\
\hline & $\mathrm{H}$ & 1.9981 & 6.0427 & -2.499 \\
\hline
\end{tabular}

$5 c$

$\begin{array}{rlrrr}1 & \mathrm{C} & -1.0275 & -2.8478 & -1.4449 \\ 2 & \mathrm{C} & 0.2873 & -3.1469 & -2.0471 \\ 3 & \mathrm{C} & 1.0828 & -4.3738 & -1.4344 \\ 4 & \mathrm{H} & 2.1605 & -4.2196 & -1.3522 \\ 5 & \mathrm{H} & 0.6025 & -4.8433 & -0.5737 \\ 6 & \mathrm{C} & 0.4706 & -4.5122 & -2.7705 \\ 7 & \mathrm{H} & 1.1189 & -4.4875 & -3.6461 \\ 8 & \mathrm{H} & -0.4375 & -5.1071 & -2.8681 \\ 9 & \mathrm{C} & 1.0941 & -1.9984 & -2.5087 \\ 10 & \mathrm{~N} & 0.9487 & -0.7461 & -2.1695 \\ 11 & \mathrm{Cu} & -0.3549 & -0.0544 & -0.8446 \\ 12 & \mathrm{~N} & -1.4443 & -1.6970 & -0.9839 \\ 13 & \mathrm{O} & 0.7073 & 1.6580 & -0.6671 \\ 14 & \mathrm{C} & 0.4839 & 2.6333 & 0.0714 \\ 15 & \mathrm{~N} & -0.5507 & 2.7789 & 1.0058 \\ 16 & \mathrm{C} & -1.5405 & 1.8193 & 1.2311 \\ 17 & \mathrm{O} & -1.5975 & 0.7554 & 0.5525 \\ 18 & \mathrm{O} & 2.0859 & -2.2728 & -3.3651 \\ 19 & \mathrm{C} & 2.7935 & -0.9880 & -3.6594 \\ 20 & \mathrm{H} & 3.8167 & -1.0975 & -3.2795 \\ 21 & \mathrm{H} & 2.7980 & -0.8757 & -4.7498 \\ 22 & \mathrm{C} & 1.9551 & 0.0784 & -2.9152 \\ 23 & \mathrm{H} & 2.5517 & 0.6701 & -2.2120 \\ 24 & \mathrm{H} & 1.4325 & 0.7618 & -3.5989 \\ 25 & \mathrm{C} & -2.8085 & -1.8865 & -0.3957 \\ 26 & \mathrm{H} & -2.7413 & -1.8079 & 0.6982 \\ 27 & \mathrm{H} & -3.4975 & -1.1195 & -0.7710 \\ 28 & \mathrm{C} & -3.1862 & -3.3055 & -0.8685 \\ 29 & \mathrm{H} & -3.8649 & -3.3130 & -1.7317 \\ 30 & \mathrm{H} & -3.5563 & -3.9693 & -0.0800 \\ 31 & \mathrm{O} & -1.8887 & -3.8700 & -1.3491 \\ 32 & \mathrm{O} & 1.2676 & 3.7024 & 0.0457 \\ 33 & \mathrm{C} & 0.7242 & 4.7702 & 0.9225 \\ 34 & \mathrm{H} & 0.3546 & 5.5637 & 0.2591 \\ 35 & \mathrm{H} & 1.5558 & 5.1352 & 1.5368 \\ 36 & \mathrm{C} & -0.3952 & 4.0782 & 1.7369 \\ 37 & \mathrm{H} & -0.0962 & 3.8874 & 2.7778 \\ 38 & \mathrm{H} & -1.3295 & 4.6532 & 1.7102 \\ 39 & \mathrm{C} & -2.5143 & 2.1065 & 2.2930 \\ 40 & \mathrm{H} & -2.3575 & 2.9917 & 2.9094 \\ 41 & \mathrm{C} & -3.5871 & 1.3157 & 2.5238 \\ 42 & \mathrm{H} & -4.2939 & 1.5554 & 3.3223 \\ 43 & \mathrm{H} & -3.7854 & 0.4260 & 1.9239\end{array}$




\begin{tabular}{rlrrr}
1 & $\mathrm{C}$ & -1.5666 & -1.9222 & 1.3524 \\
2 & $\mathrm{C}$ & -1.1939 & -2.5197 & 2.6738 \\
3 & $\mathrm{C}$ & -1.4279 & -4.1160 & 2.7292 \\
4 & $\mathrm{C}$ & -2.5475 & -3.9482 & 3.7931 \\
5 & $\mathrm{H}$ & -2.3207 & -4.4150 & 4.7597 \\
6 & $\mathrm{H}$ & -3.5362 & -4.2873 & 3.4601 \\
7 & $\mathrm{H}$ & -1.7527 & -4.5164 & 1.7614 \\
8 & $\mathrm{H}$ & -0.5316 & -4.6519 & 3.0636 \\
9 & $\mathrm{C}$ & -2.3666 & -2.4052 & 3.7761 \\
10 & $\mathrm{H}$ & -2.0127 & -1.9584 & 4.7127 \\
11 & $\mathrm{H}$ & -3.2243 & -1.8345 & 3.4006 \\
12 & $\mathrm{C}$ & 0.1449 & -2.1053 & 3.2010 \\
13 & $\mathrm{~N}$ & 1.0340 & -1.3048 & 2.6778 \\
14 & $\mathrm{Cu}$ & 0.9098 & -0.3628 & 0.9497 \\
15 & $\mathrm{~N}$ & -0.8853 & -1.1101 & 0.5919 \\
16 & $\mathrm{O}$ & 0.9756 & 0.4582 & -0.8967 \\
17 & $\mathrm{C}$ & 1.7906 & 1.2862 & -1.3386 \\
18 & $\mathrm{~N}$ & 2.9337 & 1.7879 & -0.6974 \\
19 & $\mathrm{C}$ & 3.3232 & 1.4374 & 0.5973 \\
20 & $\mathrm{O}$ & 2.6175 & 0.6764 & 1.3170 \\
21 & $\mathrm{O}$ & 0.4635 & -2.6360 & 4.3888 \\
22 & $\mathrm{C}$ & 1.8619 & -2.2256 & 4.7057 \\
23 & $\mathrm{H}$ & 2.4832 & -3.1254 & 4.6065 \\
24 & $\mathrm{H}$ & 1.8656 & -1.8676 & 5.7413 \\
25 & $\mathrm{C}$ & 2.1651 & -1.1447 & 3.6496 \\
26 & $\mathrm{H}$ & 3.1259 & -1.3021 & 3.1465 \\
27 & $\mathrm{H}$ & 2.1369 & -0.1263 & 4.0629 \\
28 & $\mathrm{C}$ & -1.6567 & -0.8787 & -0.6771 \\
29 & $\mathrm{H}$ & -1.7303 & 0.1940 & -0.8895 \\
30 & $\mathrm{H}$ & -1.1331 & -1.3685 & -1.5099 \\
31 & $\mathrm{C}$ & -3.0237 & -1.5197 & -0.3641 \\
32 & $\mathrm{H}$ & -3.8105 & -0.7904 & -0.1306 \\
33 & $\mathrm{H}$ & -3.3743 & -2.2370 & -1.1144 \\
34 & $\mathrm{O}$ & -2.7708 & -2.2884 & 0.8929 \\
35 & $\mathrm{O}$ & 1.6495 & 1.8054 & -2.5505 \\
36 & $\mathrm{C}$ & 2.6652 & 2.8666 & -2.7812 \\
37 & $\mathrm{H}$ & 3.0861 & 2.6975 & -3.7793 \\
38 & $\mathrm{H}$ & 2.1313 & 3.8259 & -2.7419 \\
39 & $\mathrm{C}$ & 3.6847 & 2.6852 & -1.6346 \\
40 & $\mathrm{H}$ & 3.9261 & 3.6402 & -1.1507 \\
41 & $\mathrm{H}$ & 4.6055 & 2.1853 & -1.9696 \\
42 & $\mathrm{C}$ & 4.5937 & 1.9941 & 1.0904 \\
43 & $\mathrm{H}$ & 5.2393 & 2.5130 & 0.3805 \\
46 & $\mathrm{C}$ & 4.9676 & 1.8799 & 2.3792 \\
& $\mathrm{H}$ & 5.9164 & 2.2973 & 2.7262 \\
& & & & \\
\hline 1
\end{tabular}

$\begin{array}{rrrrr}1 & \mathrm{C} & 0.1777 & 2.5015 & 2.1300 \\ 2 & \mathrm{C} & 1.1607 & 2.5450 & 3.2730 \\ 3 & \mathrm{C} & 0.3846 & 2.7886 & 4.6599 \\ 4 & \mathrm{C} & 1.1243 & 3.9555 & 5.3449 \\ 5 & \mathrm{C} & 1.5809 & 4.8450 & 4.1769 \\ 6 & \mathrm{H} & 0.7318 & 5.4196 & 3.7760 \\ 7 & \mathrm{H} & 2.3664 & 5.5604 & 4.4601 \\ 8 & \mathrm{H} & 0.4674 & 4.4783 & 6.0551 \\ 9 & \mathrm{H} & 1.9941 & 3.5804 & 5.9061 \\ 10 & \mathrm{H} & -0.6509 & 3.0722 & 4.4289 \\ 11 & \mathrm{H} & 0.3528 & 1.8696 & 5.2621 \\ 12 & \mathrm{C} & 2.0950 & 3.8440 & 3.1221 \\ 13 & \mathrm{H} & 2.0688 & 4.2315 & 2.0940 \\ 14 & \mathrm{H} & 3.1305 & 3.5519 & 3.3427 \\ 15 & \mathrm{C} & 2.0121 & 1.3077 & 3.3950 \\ 16 & \mathrm{~N} & 2.0221 & 0.2249 & 2.6669 \\ 17 & \mathrm{Cu} & 0.8789 & -0.1803 & 1.1125 \\ 18 & \mathrm{~N} & -0.0427 & 1.5598 & 1.2544 \\ 19 & \mathrm{O} & -0.4146 & -0.8135 & -0.3073 \\ 20 & \mathrm{C} & -0.3278 & -1.8204 & -1.0310 \\ 21 & \mathrm{~N} & 0.6500 & -2.8280 & -0.9839 \\ 22 & \mathrm{C} & 1.7231 & -2.8329 & -0.0887 \\ 23 & \mathrm{O} & 1.9221 & -1.8712 & 0.7045 \\ 24 & \mathrm{O} & 2.8823 & 1.3335 & 4.4144 \\ 25 & \mathrm{C} & 3.5287 & -0.0084 & 4.4882 \\ 26 & \mathrm{H} & 3.1176 & -0.5068 & 5.3762 \\ 27 & \mathrm{H} & 4.6055 & 0.1576 & 4.6047 \\ 28 & \mathrm{C} & 3.1187 & -0.6728 & 3.1613 \\ 29 & \mathrm{H} & 3.9315 & -0.6813 & 2.4211 \\ 30 & \mathrm{H} & 2.7459 & -1.6943 & 3.2974 \\ 31 & \mathrm{C} & -1.1919 & 1.9752 & 0.3797 \\ 32 & \mathrm{H} & -2.0684 & 1.3583 & 0.6235 \\ 33 & \mathrm{H} & -0.9415 & 1.8309 & -0.6772 \\ 34 & \mathrm{C} & -1.3763 & 3.4581 & 0.7519 \\ 35 & \mathrm{H} & -2.4052 & 3.7451 & 0.9947 \\ 36 & \mathrm{H} & -0.9434 & 4.1534 & 0.0205 \\ 37 & \mathrm{O} & -0.5727 & 3.6049 & 2.0039 \\ 38 & \mathrm{O} & -1.2222 & -2.0575 & -1.9818 \\ 39 & \mathrm{C} & -0.8148 & -3.2311 & -2.7986 \\ 40 & \mathrm{H} & -1.7049 & -3.8582 & -2.9267 \\ 41 & \mathrm{H} & -0.4737 & -2.8359 & -3.7650 \\ 42 & \mathrm{C} & 0.3104 & -3.9020 & -1.9772 \\ 43 & \mathrm{H} & -0.0397 & -4.7996 & -1.4468 \\ 44 & \mathrm{H} & 1.1804 & -4.1440 & -2.6006 \\ 45 & \mathrm{C} & 2.6126 & -4.0042 & -0.1093 \\ 46 & \mathrm{H} & 2.3102 & -4.8695 & -0.7008 \\ 47 & \mathrm{C} & 3.7735 & -4.0294 & 0.5730 \\ 48 & \mathrm{H} & 4.4195 & -4.9108 & 0.5457 \\ 49 & \mathrm{H} & 4.1135 & -3.1742 & 1.1600 \\ & & & & \end{array}$




\begin{tabular}{|c|c|c|c|c|}
\hline 1 & $\mathrm{C}$ & 1.6141 & -1.1112 & 0.8697 \\
\hline 2 & $\mathrm{C}$ & 2.4551 & -0.0095 & 1.4897 \\
\hline 3 & $\mathrm{C}$ & 3.9516 & -0.1001 & 0.9331 \\
\hline 4 & $\mathrm{C}$ & 4.8261 & -1.1899 & 1.5822 \\
\hline 5 & $\mathrm{C}$ & 4.8058 & -1.1033 & 3.1183 \\
\hline 6 & $\mathrm{H}$ & 5.2428 & -0.1411 & 3.4430 \\
\hline 7 & $\mathrm{H}$ & 5.4377 & -1.8960 & 3.5469 \\
\hline 8 & $\mathrm{C}$ & 3.3700 & -1.2293 & 3.6571 \\
\hline 9 & $\mathrm{H}$ & 3.3601 & -1.1130 & 4.7531 \\
\hline 10 & $\mathrm{H}$ & 2.9756 & -2.2341 & 3.4445 \\
\hline 11 & $\mathrm{H}$ & 5.8511 & -1.0464 & 1.2031 \\
\hline 12 & $\mathrm{H}$ & 4.5087 & -2.1918 & 1.2563 \\
\hline 13 & $\mathrm{H}$ & 4.4097 & 0.8782 & 1.1361 \\
\hline 14 & $\mathrm{H}$ & 3.9132 & -0.2201 & -0.1625 \\
\hline 15 & $\mathrm{C}$ & 2.4393 & -0.1433 & 3.0821 \\
\hline 16 & $\mathrm{H}$ & 2.7602 & 0.8317 & 3.4751 \\
\hline 17 & $\mathrm{H}$ & 1.3980 & -0.2981 & 3.4113 \\
\hline 18 & $\mathrm{C}$ & 1.9396 & 1.3814 & 1.1508 \\
\hline 19 & $\mathrm{~N}$ & 0.9231 & 1.7535 & 0.4220 \\
\hline 20 & $\mathrm{Cu}$ & -0.3536 & 0.6058 & -0.5359 \\
\hline 21 & $\mathrm{~N}$ & 0.5635 & -1.0209 & 0.0970 \\
\hline 22 & $\mathrm{O}$ & -1.5638 & -0.4589 & -1.7616 \\
\hline 23 & $\mathrm{C}$ & -2.5570 & -0.0708 & -2.4024 \\
\hline 24 & $\mathrm{~N}$ & -3.0618 & 1.2340 & -2.4918 \\
\hline 25 & $\mathrm{C}$ & -2.5130 & 2.3252 & -1.8162 \\
\hline 26 & $\mathrm{O}$ & -1.5367 & 2.1898 & -1.0277 \\
\hline 27 & $\mathrm{O}$ & 2.6534 & 2.3779 & 1.6924 \\
\hline 28 & $\mathrm{C}$ & 2.1221 & 3.6636 & 1.1646 \\
\hline 29 & $\mathrm{H}$ & 2.8765 & 4.0543 & 0.4686 \\
\hline 30 & $\mathrm{H}$ & 2.0019 & 4.3384 & 2.0194 \\
\hline 31 & $\mathrm{C}$ & 0.8062 & 3.2486 & 0.4816 \\
\hline 32 & $\mathrm{H}$ & 0.7028 & 3.6634 & -0.5275 \\
\hline 33 & $\mathrm{H}$ & -0.0822 & 3.5120 & 1.0728 \\
\hline 34 & $\mathrm{C}$ & 0.1611 & -2.4010 & -0.3397 \\
\hline 35 & $\mathrm{H}$ & -0.9146 & -2.5505 & -0.1948 \\
\hline 36 & $\mathrm{H}$ & 0.3941 & -2.5212 & -1.4072 \\
\hline 37 & $\mathrm{C}$ & 1.0161 & -3.3018 & 0.5680 \\
\hline 38 & $\mathrm{H}$ & 0.4614 & -3.7300 & 1.4135 \\
\hline 39 & $\mathrm{H}$ & 1.5785 & -4.0809 & 0.0418 \\
\hline 40 & $\mathrm{O}$ & 2.0164 & -2.3568 & 1.1520 \\
\hline 41 & $\mathrm{O}$ & -3.2806 & -0.9193 & -3.1217 \\
\hline 42 & $\mathrm{C}$ & -4.4744 & -0.2367 & -3.6763 \\
\hline 43 & $\mathrm{H}$ & -4.5445 & -0.5191 & -4.7333 \\
\hline 44 & $\mathrm{H}$ & -5.3414 & -0.6149 & -3.1174 \\
\hline 45 & $\mathrm{C}$ & -4.2117 & 1.2688 & -3.4506 \\
\hline 46 & $\mathrm{H}$ & -3.9109 & 1.7842 & -4.3746 \\
\hline 47 & $\mathrm{H}$ & -5.0792 & 1.7695 & -3.0023 \\
\hline 48 & $\mathrm{C}$ & -3.1255 & 3.6454 & -2.0603 \\
\hline 49 & $\mathrm{H}$ & -3.8686 & 3.7270 & -2.8547 \\
\hline 50 & $\mathrm{C}$ & -2.7969 & 4.7324 & -1.3352 \\
\hline 51 & $\mathrm{H}$ & -2.0704 & 4.6833 & -0.5220 \\
\hline 52 & $\mathrm{H}$ & -3.2647 & 5.6999 & -1.5357 \\
\hline
\end{tabular}

